\title{
A benchmark-based evaluation of search-based crash reproduction
}

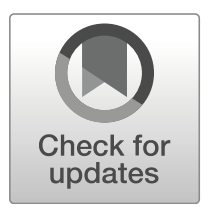

\author{
Mozhan Soltani $^{1,2} \cdot$ Pouria Derakhshanfar $^{1} \cdot$ Xavier Devroey $^{1}$ (D) $\cdot$ Arie van Deursen ${ }^{1}$
}

Published online: 29 August 2019

(C) The Author(s) 2019

\begin{abstract}
Crash reproduction approaches help developers during debugging by generating a test case that reproduces a given crash. Several solutions have been proposed to automate this task. However, the proposed solutions have been evaluated on a limited number of projects, making comparison difficult. In this paper, we enhance this line of research by proposing JCrashPack, an extensible benchmark for Java crash reproduction, together with ExRunner, a tool to simply and systematically run evaluations. JCrashPack contains 200 stack traces from various Java projects, including industrial open source ones, on which we run an extensive evaluation of EvoCrash, the state-of-the-art tool for search-based crash reproduction. EvoCrash successfully reproduced $43 \%$ of the crashes. Furthermore, we observed that reproducing NullPointerException, IllegalArgumentException, and IllegalStateException is relatively easier than reproducing ClassCastException, ArrayIndexOutOfBoundsException and StringIndexOutOfBoundsException. Our results include a detailed manual analysis of EvoCrash outputs, from which we derive 14 current challenges for crash reproduction, among which the generation of input data and the handling of abstract and anonymous classes are the most frequents. Finally, based on those challenges, we discuss future research directions for search-based crash reproduction for Java.
\end{abstract}

Keywords Crash reproduction · Empirical evaluation $\cdot$ Search-based software testing

\section{Introduction}

Software crashes commonly occur in operating environments and are reported to developers for inspection. When debugging, reproducing a reported crash is among the tasks a

Communicated by: Mark Harman

Mozhan Soltani and Pouria Derakhshanfar contributed equally to this work

This research was partially funded by the EU Project STAMP ICT-16-10 No.731529 and the NIRICT 3TU.BSR (Big Software on the Run) project.

Xavier Devroey

x.d.m.devroey@tudelft.nl

Extended author information available on the last page of the article. 
developer needs to do in order to identify the conditions under which the reported crash is triggered (Zeller 2009). To help developers in this process, various automated techniques have been suggested. These techniques typically either use program runtime data (Artzi et al. 2008; Clause and Orso 2007; Narayanasamy et al. 2005; Steven et al. 2000; Gómez et al. 2016; Bell et al. 2013; Cao et al. 2014; Rößler et al. 2013) or crash stack traces (Bianchi et al. 2017; Soltani et al. 2017; Nayrolles et al. 2017; Xuan et al. 2015; Chen and Kim 2015) to generate a test case that triggers the reported crash.

When available, runtime data offer more information to accurately reproduce a crash. However, it also raises various concerns (for instance, privacy violation) and may induce a significant overhead during data collection (Chen and Kim 2015; Nayrolles et al. 2017; Rößler et al. 2013). Instead, we focus on crash reproduction based on a crash stack trace generated by a failing system. Practically, those stack traces are collected from the logs produced by the operating environment or reported by users in an issue tracking system. Various auromated crash stack trace-based reproduction approaches have been implemented and evaluated on different benchmarks (Soltani et al. 2017; Nayrolles et al. 2017; Xuan et al. 2015; Chen and Kim 2015). However, those benchmarks contains a limited number of crashes and associated stack traces.

In a recent study, we presented a search-based approach called EvoCrash, which applies a guided genetic algorithm to search for a crash reproducing test case (Soltani et al. 2017), and demonstrated its relevance for debugging (Soltani et al. 2018). We conducted an empirical evaluation on 54 crashes from commonly used utility libraries to compare EvoCrash with state-of-the-art techniques for crash reproduction (Soltani et al. 2017). This was enough to show that the search-based crash reproduction outperformed other approaches based on backward symbolic execution (Chen and Kim 2015), test case mutation (Xuan et al. 2015), and model-checking (Nayrolles et al. 2017), evaluated on smaller benchmarks.

However, all those crashes benchmarks were not selected to reflect challenges that are likely to occur in real life stack traces, raising threats to external validity. Thus the questions whether the selected applications and crashes were sufficiently representative, if EvoCrash will work in other contexts, and what limitations are still there to address, remained unanswered.

The goal of this paper is to facilitate sound empirical evaluation on automated crash reproduction approaches. To that end, we devise a new benchmark of real-world crashes, called JCrashPack. It contains 200 crashes from seven actively maintained open-source and industrial projects. These projects vary in their domain application and include an enterprise wiki application, a distributed RESTful search engine, several popular APIs, and a mocking framework for unit testing Java programs. JCrashPack is extensible, and can be used for large-scale evaluation and comparison of automated crash reproduction techniques for Java programs.

To illustrate the use of JCrashPack, we adopt it to extend the reported evaluation on EvoCrash (Soltani et al. 2017) and identify the areas where the approach can be improved. In this experience report, we provide an account of the cases that were successfully reproduced by EvoCrash ( 87 crashes out of 200 ). We also analyze all failed reproductions and distill 14 categories of research and engineering limitations that negatively affected reproducing crashes in our study. Some of those limitations are in line with challenges commonly reported for search-based structural software testing in the community (Xiao et al. 2011; McMinn 2011; Fraser and Arcuri 2014b) and others are specific to search-based crash reproduction.

Our categorization of challenges indicates that environmental dependencies, code complexity, and limitations of automated input data generation often hinder successful crash 
reproduction. In addition, stack frames (i.e., lines in a stack trace), pointing to varying types of program elements, such as interfaces, abstract classes, and anonymous objects, influence the extent to which a stack trace-based approach to crash reproduction is effective.

Finally, we observe that the percentage of successfully reproduced crashes drops from $85 \%$ (46 crashes out of 54 reported by Soltani et al. (2018)) to $43 \%$ (87 out of 200) when evaluating crashes that are from industrial projects. In our observations, generating input data for microservices, and unit testing for classes with environmental dependencies, which may frequently exist in enterprise applications, are among the major reasons for the observed drop in the reproduction rate. These results are consistent with the paradigm shift to context-based software engineering research that has been proposed by Briand et al. (2017).

The key contributions of our paper are:

- JCrashPack, ${ }^{1}$ a carefully composed benchmark of 200 crashes, as well as their correct system version and its libraries, from seven real-world Java projects, together with an account of our manual analysis on the characteristics of the selected crashes and their constituting frames, including size of the stack traces, complexity measures, and identification of buggy and fixed versions.

- ExRunner, ${ }^{2}$ a Python library for automatically running experiments with crash reproduction tools in Java.

- Empirical evidence, ${ }^{3}$ demonstrating the effectiveness of search-based crash reproduction on real world crashes taken from JCrashPack.

- The identification of 14 categories of research and engineering challenges for searchbased crash reproduction that need to be addressed in order to facilitate uptake in practice of crash reproduction research.

The remainder of the paper is structured as follows: Section 2 presents background on crash reproduction. Sections 3 to 5 describe the design protocol for the benchmark, the resulting benchmark JCrashPack, as well as the ExRunner tool to run experiments on JCrashPack. Sections 6 to 8 cover the experimental setup for the EvoCrash evaluation, the results from our evaluation, and the results challenges that we identified through our evaluation. Sections 9 to 12 provide a discussion of our results and future research directions, an analysis of the threats to validity, and a summary of our overall conclusions.

\section{Background and Related Work}

\subsection{Crash Reproduction}

Crash reproduction approaches can be divided into three categories, based on the kind of data used for crash reproduction: record-replay approaches record data from the running program; post-failure approaches collect data from the crash, like a memory dump; and stack-trace based post-failure use only the stack trace produced by the crash. We briefly describe each category hereafter.

\footnotetext{
${ }^{1}$ Available at https://github.com/STAMP-project/JCrashPack.

${ }^{2}$ Available at https://github.com/STAMP-project/ExRunner

${ }^{3}$ A replication package for EvoCrash results, their automated analysis, and the results of our manual analysis is available at https://github.com/STAMP-project/EvoCrash-JCrashPack-application.
} 
Table 1 The crash stack trace for Apache Ant-49755

java.lang.NullPointerException:

Level

Frame

2

3

at org.apache.tools.ant.util.FileUtils.createTempFile(FileUtils.java:888)

at org.apache.tools.ant.taskdefs.TempFile.execute(TempFile.java:158)

at org.apache.tools.ant.UnknownElement.execute(UnknownElement.java:291)

Record-Replay Approaches These approaches record the program runtime data and use them during crash reproduction. The main limitation is the availability of the required data. Monitoring software execution may violate privacy by collecting sensitive data, the monitoring process can be an expensive task for the large scale software and may induce a significant overhead (Chen and Kim 2015; Nayrolles et al. 2017; Rößler et al. 2013). Tools like ReCrash (Artzi et al. 2008), ADDA (Clause and Orso 2007), Bugnet (Narayanasamy et al. 2005), jRapture (Steven et al. 2000), MoTiF (Gómez et al. 2016), Chronicler (Bell et al. 2013), and SymCrash (Cao et al. 2014) fall in this category.

Post-Failure Approaches Tools from this category use the software data collected directly after the occurrence of a failure. For instance, RECORE (Rößler et al. 2013) applies a search-based approach to reproduce a crash. RECORE requires both a stack trace and a core dump, produced by the system when the crash happened, to guide the search. Although these tools limit the quantity of monitored and recorded data, the availability of such data still represents a challenge. For instance, if the crash is reported trough an issue tracking system or if the core dump contains sensitive data. Other post-failure approaches inlcude: DESCRY (Yu et al. 2017), and other tools by Weeratunge et al. (2010), Leitner et al. (2007, 2009), or Kifetew et al. (2013, 2014).

Stack-trace based post-failure Recent studies in crash reproduction (Bianchi et al. 2017; Soltani et al. 2017; Nayrolles et al. 2017; Xuan et al. 2015; Chen and Kim 2015) focuses on utilizing data only from a given crash stack trace to enhance the practical application of the tools. For instance, in contrast to the previously introduced approaches, EvoCrash only considers the stack trace (usually provided when a bug is reported in an issue tracker) and a distance, similar to the one described by Rossler et al. (2013), to guide the search. Table 1 illustrates an example of a crash stack trace from Apache $\mathrm{Ant}^{4}$ (Apache 2017a) which is comprised of a crash type (java.lang. NullPointerException) and a stack of frames pointing to all method calls that were involved in the execution when the crash happened. From a crash stack frame, we can retrieve information about: the crashing method, the line number in the method where the crash happened, and the fully qualifying name of the class where the crashing method is declared.

The state of the research in crash reproduction (Zamfir and Candea 2010; Jin and Orso 2012; Bianchi et al. 2017; Soltani et al. 2017; Nayrolles et al. 2017; Xuan et al. 2015; Chen and Kim 2015) aims at generating test code that, once executed, produces a stack trace that is as similar to the original one as possible. They, however, differ in their means to achieve this task: for instance, ESD (Zamfir and Candea 2010) and BugRedux (Jin and Orso 2012) use forward symbolic execution; STAR (Chen and Kim 2015) applies optimized

\footnotetext{
${ }^{4}$ ANT-49755: https://bz.apache.org/bugzilla/show_bug.cgi?id=49755
} 
backward symbolic execution and a novel technique for method sequence composition; JCHARMING (Nayrolles et al. 2017) applies model checking; MuCrash (Xuan et al. 2015) is based on exploiting existing test cases that are written by developers, and mutating them until they trigger the target crash; and Concrash (Bianchi et al. 2017) focuses on reproducing concurrency failures that violate thread-safety of a class by using search pruning strategies.

\subsection{Search-Based Crash Reproduction with EvoCrash}

Search-based algorithms have been increasingly used for software engineering problems since they are shown to suite complex, non-linear problems, with multiple optimization objectives which may be in conflict or competing (Harman et al. 2012). Recently, Soltani et al. $(2017,2018)$ introduced a search-based approach to crash reproduction, called EvoCrash. EvoCrash applies a guided genetic algorithm to search for a unit test that reproduces the target crash. To generate the unit tests, EvoCrash relies on a search-based test generator called EvoSuite (Fraser and Arcuri 2013b).

EvoCrash takes as input a stack trace with one of its frames set as the target frame. The target frame is composed of a target class, the class to which the exception has been propagated, a target method, the method in that class, and a target line, the line in that method where the exception has been propagated. Then, it seeks to generate a unit test which replicates the given stack trace from the target frame (at level $n$ ) to the deepest frame (at level 1). For instance, if we pass the stack trace in Table 1 as the given trace and indicate the second frame as the target frame (level 2), the output of EvoCrash will be a unit test for the class TempFile which replicates first two frames of the given stack trace with the same type of the exception (NullPointerException).

\subsubsection{Guided Genetic Algorithm}

The search process in EvoCrash begins by randomly generating unit tests for the target frame. In this phase, called guided initialization, the target method corresponding to the selected frame (i.e., the failing method to which the exception is propagated) is injected in every randomly generated unit test. During subsequent phases of the search, guided crossover and guided mutation, standard evolutionary operations are applied to the unit tests. However, applying these operations involves the risk of losing the injected failing method. Therefore, the algorithm ensures that only unit tests with the injected failing method call remain in the evolution loop. If the generated test by crossover does not contain the failing method, the algorithm replaces it with one of its parents. Also, if after a mutation, the resulting test does not contain the failing method, the algorithm redoes the mutation until the the failing method is added to the test again. The search process continues until either the search budget is over or a crash reproducing test case is found.

To evaluate the generated tests, EvoCrash applies the following weighted sum fitness function (Soltani et al. 2018) to a generated test $t$ :

$$
f(t)= \begin{cases}3 \times d_{s}(t)+2 \times \max \left(d_{\text {except }}\right)+\max \left(d_{\text {trace }}\right) & \text { ifthelineisnotreached } \\ 3 \times \min \left(d_{s}\right)+2 \times d_{\text {except }}(t)+\max \left(d_{\text {trace }}\right) & \text { ifthelineisreached } \\ 3 \times \min \left(d_{s}\right)+2 \times \min \left(d_{\text {except }}\right)+d_{\text {trace }}(t) & \text { iftheexceptionisthrown }\end{cases}
$$

Where:

- $\quad d_{s} \in[0,1]$ indicates the distance between the execution of $t$ and the target statement $s$ located at the target line. This distance is computed using the approach level, measuring 
the minimum number of control dependencies between the path of the code executed by $t$ and $s$, and normalized branch distance, scoring how close $t$ is to satisfying the branch condition for the branch on whichs is directly control dependent (McMinn 2004). If the target line is reached by the test case, $d_{l}(t)$ equals to 0.0 ;

$-d_{\text {except }}(t) \in\{0,1\}$ indicates if the target exception is thrown $\left(d_{e}=0\right)$ or not $\left(d_{e}=1\right)$;

- $d_{\text {trace }}(t) \in[0,1]$ indicates the similarity of the input stack trace and the one generated by $t$ by looking at class names, methods names and line numbers;

- $\max (\cdot)$ denotes the maximum possible value for the function.

Since the stack trace similarity is relevant only if the expected exception is thrown by $t$, and the check whether the expected exception is thrown or not is relevant only if the target line where the exception propagates is reached, $d_{\text {except }}$ and $d_{\text {trace }}$ are computed only upon the satisfaction of two constraints: the target exception has to be thrown in the target line $s$ and the stack trace similarity should be computed only if the target exception is actually thrown.

Unlike other stack trace similarity measures (e.g., Rößler et al. 2013), Soltani et al. (2018) do not require two stack traces to share the same common prefix to avoid rejecting stack traces where the difference is only in one intermediate frame. Instead, for each frame, $d_{\text {trace }}(t)$ looks at the closest frame and compute a distance value. Formally, for an original stack trace $S *$ and a test case $t$ producing a stack trace $S, d_{\text {trace }}(t)$ is defined as follows:

$$
d_{\text {trace }}(t)=\varphi\left(\sum_{f * \in S *} \min \{\operatorname{diff}(f *, f): f \in S\}\right)
$$

Where $\varphi(x)=x /(x+1)$ is a normalization function (McMinn 2004) and $\operatorname{diff}(f *, f)$ measures the difference between two frames as follows:

$$
\operatorname{diff}(f *, f)= \begin{cases}3 & \text { iftheclassesaredifferent } \\ 2 & \text { iftheclassesareequalbutthemethodsaredifferent } \\ \varphi(|l *-l|) & \text { otherwise }\end{cases}
$$

Where $l$ (resp. $l *$ ) is the line number of the frame $f$ (resp. $f *$ ).

Each of the three components if the fitness function defined in (1) ranges from 0.0 to 1.0 , the overall fitness value for a given test case ranges from 0.0 (crash is fully reproduced) to 6.0 (no test was generated), depending on the conditions it satisfies.

\subsubsection{Comparison with the State-of-the-art}

Crash Reproduction Tools Table 2 presents the number of crashes used in the benchmarks used to evaluated stack-trace based post-failure crash reproduction tools as well as their crash reproduction rates. EvoCrash has been evaluated on various crashes reported in other studies and has the highest reproduction rate.

EvoSuite Table 2 also reports the comparison of EvoCrash with EvoSuite, using exception coverage as the primary objective, applied by Soltani et al. (2018). All the crashes reproduced by EvoSuite could also be reproduced by EvoCrash on average $170 \%$ faster and with a higher reproduction rate. 
Table 2 The number of crashes used in each crash reproduction tool experiment, the gained reproduction by them, and the involved projects

\begin{tabular}{|c|c|c|c|}
\hline Tool & Reproduced/Total & Rate & Projects \\
\hline EvoCrash (Soltani et al. 2017, 2018) & $46 / 54$ & $85 \%$ & $\begin{array}{l}\text { Apache Commons Collections } \\
\text { Apache Ant } \\
\text { Apache Log } 4 \mathrm{j} \\
\text { ActiveMQ } \\
\text { DnsJava } \\
\text { JFreeChart }\end{array}$ \\
\hline EvoSuite (Soltani et al. 2018) & $18 / 54$ & $33 \%$ & $\begin{array}{l}\text { Apache Commons Collections } \\
\text { Apache Ant } \\
\text { Apache Log } 4 \mathrm{j} \\
\text { ActiveMQ } \\
\text { DnsJava } \\
\text { JFreeChart }\end{array}$ \\
\hline STAR (Chen and Kim 2015) & $30 / 51$ & $59 \%$ & $\begin{array}{l}\text { Apache Commons Collections } \\
\text { Apache Ant } \\
\text { Apache Log } 4 \mathrm{j}\end{array}$ \\
\hline MuCrash (Xuan et al. 2015) & $8 / 12$ & $66 \%$ & Apache Commons Collections \\
\hline JCharming(Nayrolles et al. 2017) & $8 / 12$ & $66 \%$ & $\begin{array}{l}\text { Apache Ant } \\
\text { Apache Log4j } \\
\text { ActiveMQ } \\
\text { DnsJava } \\
\text { JFreeChart }\end{array}$ \\
\hline
\end{tabular}

\section{Benchmark Design}

Benchmarking is a common practice to assess a new technique and compare it to the state of the art (Sim et al. 2003). For instance, SF110 (Fraser and Arcuri 2014b) is a sample of 100 Java projects from SourceForge, and 10 popular Java projects from GitHub, that may be used to assess (search based) test case selection techniques. In the same way, Defects4J (Just et al. 2014) is a collection of bugs coming from popular open-source projects: for each bug, a buggy and a fixed version of the projects, as well as bug revealing test case, are provided. Defects4J is aimed to assess various testing techniques like test case selection or fault localization.

In their previous work, Soltani et al. (2017), Xuan et al. (2015), and Chen and Kim (2015) used Apache Commons Collections (Apache 2017b), Apache Ant (Apache 2017a), and Apache Log4j (Apache 2017c) libraries. In addition to Apache Ant and Apache Log4j, Nayrolles et al. (2017) used bug reports from 8 other open-source software.

In this paper we enhance previous efforts to build a benchmark dedicated to crash reproduction by collecting cases coming from both state of the art literature and actively maintained industrial open-source projects with well documented bug trackers. 


\subsection{Projects Selection Protocol}

As Table 2 clearly shows, current crash reproduction tools are not evaluated using a common benchmark. This hampers progress in the field as it makes it hard to compare approaches. To be able to perform analysis of the results of a crash reproduction attempt, we define the following benchmark requirements for our benchmark:

BR1, to be part of the benchmark, the projects should have openly accessible binaries, source code, and crash stack traces (in an issue tracker for instance);

BR2, they should be under active maintenance to be representative of current software engineering practices and ease communication with developers;

BR3, each stack trace should indicate the version of the project that generated the stack trace; and

BR4, the benchmark should include projects of varying size.

To best of our knowledge, there is no benchmark fulfilling those requirements. The closest benchmark is Defects $4 \mathrm{j}$. However, only $25 \%$ of the defects manifest trough a crash stack trace (BR1) and the projects are relatively small (BR4). To address those limitations, we built a new benchmark dedicated to the crash reproduction tools.

To build our benchmark, we took the following approach. First, we investigated projects collected in SF110 (Fraser and Arcuri 2014b) and Defects4J (Just et al. 2014) as state of the art benchmarks. However, as most projects in SF110 have not been updated since 2010 or earlier, we discarded them from our analysis (BR2). From Defects4J, we collected 73 cases where bugs correspond to actual crashes: i.e., the execution of the test case highlighting the bug in a given buggy version of a project generates a stack trace that is not a test case assertion failure.

As also discussed by Fraser and Arcuri (Fraser and Arcuri 2014b), to increase the representativeness of a benchmark, it is important to include projects that are popular and attractive to end-users. Additionally to Defects4J, we selected two industrial open-source projects: XWiki (2018) and Elasticsearch (Elastic 2018). XWiki is a popular enterprise wiki management system. Elasticsearch, a distributed RESTful search and analytic engine, is one of the ten most popular projects on GitHub. ${ }^{5}$ To identify the top ten popular projects from Github, we took the following approach: (i) we queried the top ten projects that had the highest number of forks; (ii) we queried the top ten projects that had the highest number of stars; (iii) we queried the top ten trending projects; and (iv) took the intersection of the three.

Four projects were shared among the above top-ten projects, namely: Java-designpatterns (Java Design Patterns 2018), Dubbo (2018), RxJava (2018), and Elasticsearch. To narrow down the scope of the study, we selected Elasticsearch, which ranked the highest among the four shared projects.

\subsection{Stack Trace Collection and Preprocessing}

For each project, we collected stack traces to be reproduced as well as the project binaries, with specific versions on which the exceptions happened.

Defects4J From the 395 buggy versions of the Defects4J projects, we kept only the bugs relevant to our crash reproduction context (73 cases), i.e., the bugs that manifest as crashes.

${ }^{5}$ This selection was performed on $26 / 10 / 2017$. 
We manually inspected the stack traces generated by the failing tests and collected those which are not JUnit assertion failures (i.e., those which are due to an exception thrown by the code under test and not by the JUnit framework). For instance, for one stack trace from the Joda-Time project:

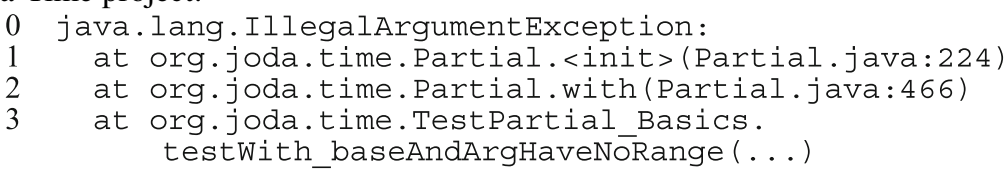

We only consider the first and second frames (lines 1 and 2). The third and following lines concern testing classes of the project, which are irrelevant for crash reproduction. They are removed from the benchmark, resulting in the following stack trace with two frames:

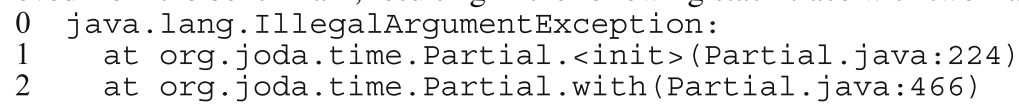

We proceeded in the same way for each Defects $4 \mathrm{~J}$ project and collected a total of 73 stack traces coming from five (out of the six) projects: JFreeChart, Commons-lang, Commonsmath, Mockito, and Joda-Time. All the stack traces generated by the Closure compiler test cases are JUnit assertion failures.

Elasticsearch Crashes for Elasticsearch are publicly reported to the issue tracker of the project on GitHub. ${ }^{6}$ Therefore, we queried the reported crashes, which were labelled as bugs, using the following string "exception is: issue label:bug". From the resulting issues (600 approx.), we manually collected the most recent ones (reported since 2016), which addressed the following: (i) the version which crashed was reported, (ii) the issue was discussed by the developers and approved as a valid crash to be fixed. The above manual process resulted in 76 crash stack traces.

XWiki XWiki is an open source project which has a public issue tracker. ${ }^{7}$ We investigated first 1000 issues which are reported for XWIK-7.2 (released in September 2015) to XWIK9.6 (released in July 2017). We selected the issues where: (i) the stack trace of the crash was included in the reported issue, and (iii) the reported issue was approved by developers as a valid crash to be fixed. Eventually, we selected a total of 51 crashes for XWIKI.

\section{The JCrashPack Benchmark}

The result of our selection protocol is a benchmark with 200 stack traces called JCrashPack. For each stack trace, based on the information from the issue tracker and the Defects $4 \mathrm{~J}$ data, we collected: the Java project in which the crash happened, the version of the project where the crash happened and (when available) the fixed version or the fixing commit reference of the project; the buggy frame (i.e., the frame in the stack trace targeting the method where the bug lays); and the Cyclomatic Complexity Number $(\mathrm{CCN})$ and the Non-Commenting Sources Statements (NCSS) of the project, presented in Fig. 1. Due to the manual effort involved in filtering, verifying and cleaning up stack traces, issues, the collection of stack traces and binaries (including the project's dependencies binaries) took about 4.5 person-months in total.

\footnotetext{
${ }^{6}$ https://github.com/elastic/elasticsearch/issues

${ }^{7}$ https://jira.Xwiki.org/browse/XWIKI/
} 


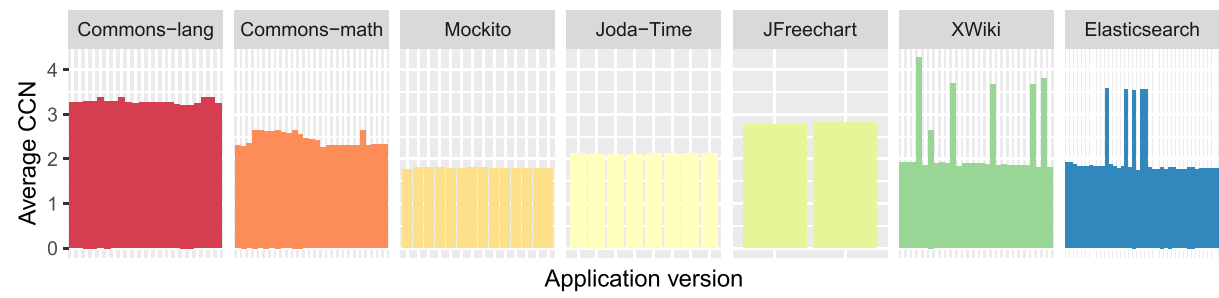

(a) Average methods Cyclomatic Complexity Number (CCN)
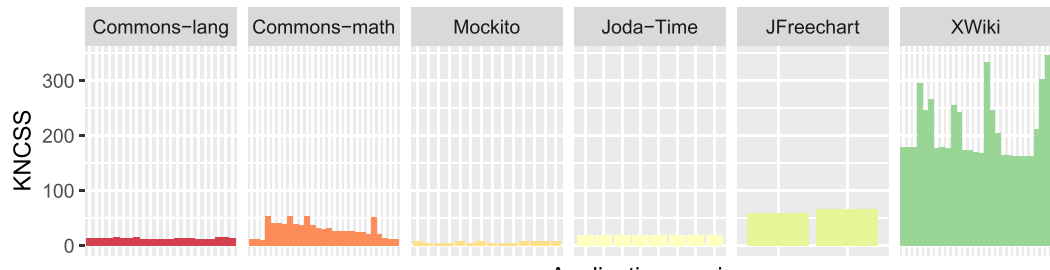

Elasticsearch

Application version

(b) Thousands of Non-Commenting Sources Statements (KNCSS)

Fig. 1 Complexity and size of the different projects

Figure 1 presents the average Cyclomatic Complexity Number (CCN) per method for each project and the Non-Commenting Sources Statements (NCSS) per project, ordered by version number, to give an idea of the complexity of a project. Also, Table 3 gives the number of versions and the average number of non-commenting source statement for each project in JCrashPack. As illustrated in the table and figure, JCrashPack contains projects of diverse complexities (the $\mathrm{CCN}$ for the least complex project is 1.77 , and for the most complex is 3.38) and sizes (the largest project has 177,840 statements, and the smallest one holds 6,060 statements on average), distributed among different versions.

Table 4 shows the distribution of stack traces per exception type for the six most common ones, the Other category denoting remaining exception types. According to this table, the included stack traces in JCrashPack covers different types of the exceptions. Also, they are varied in the size (number of frames): the smallest stack traces have one frame and the largest, a user-defined exception in Other, has 175 frames.

Table 3 The number of versions and average number of statements $(\overline{N C S S})$ for each project

\begin{tabular}{lll}
\hline Applications & Number of versions & $\overline{N C S S}$ \\
\hline Commons-lang & 22 & $13.38 \mathrm{k}$ \\
Commons-math & 27 & $29.98 \mathrm{k}$ \\
Mockito & 14 & $6.06 \mathrm{k}$ \\
Joda-Time & 8 & $19.41 \mathrm{k}$ \\
JFreechart & 2 & $63.01 \mathrm{k}$ \\
XWiki & 32 & $177.84 \mathrm{k}$ \\
Elasticsearch & 46 & $124.36 \mathrm{k}$ \\
Total & 151 & $62.01 \mathrm{k}$ \\
\hline
\end{tabular}


Table 4 Number of stack traces (st), total number of frames $(f r)$, and average number of frames $(\overline{f r})$ and standard deviation $(\sigma)$ per stack trace for the different exceptions: NullPointerException (NPE), IllegalArgumentException (IAE), ArrayIndexOutOfBoundsException (AIOOBE), ClassCastException (CCE), StringIndexOutOfBoundsException (SIOOBE), IllegalStateException (ISE), and other exceptions types (Other)

\begin{tabular}{|c|c|c|c|c|c|c|c|c|c|}
\hline Applications & & NPE & IAE & AIOOBE & $\mathrm{CCE}$ & SIOOBE & ISE & Other & Total \\
\hline \multirow[t]{4}{*}{ Commons-lang } & st & 5.0 & 3.0 & 2.0 & 0.0 & 6.0 & 0.0 & 6.0 & 22.0 \\
\hline & $f r$ & 8.0 & 3.0 & 12.0 & 0.0 & 10.0 & 0.0 & 12.0 & 45.0 \\
\hline & $\overline{f r}$ & 1.6 & 1.0 & 6.0 & & 1.7 & & 2.0 & 2.0 \\
\hline & $\sigma$ & 0.9 & 0.0 & 5.7 & & 1.0 & & 1.5 & 2.1 \\
\hline \multirow[t]{4}{*}{ Commons-math } & $s t$ & 3.0 & 3.0 & 4.0 & 2.0 & 1.0 & 0.0 & 14.0 & 27.0 \\
\hline & $f r$ & 8.0 & 7.0 & 9.0 & 11.0 & 1.0 & 0.0 & 70.0 & 106.0 \\
\hline & $\overline{f r}$ & 2.7 & 2.3 & 2.2 & 5.5 & 1.0 & & 5.0 & 3.9 \\
\hline & $\sigma$ & 0.6 & 1.5 & 2.5 & 6.4 & NA & & 3.0 & 3.0 \\
\hline \multirow[t]{4}{*}{ Mockito } & st & 2.0 & 0.0 & 2.0 & 2.0 & 0.0 & 0.0 & 8.0 & 14.0 \\
\hline & $f r$ & 3.0 & 0.0 & 12.0 & 2.0 & 0.0 & 0.0 & 48.0 & 65.0 \\
\hline & $\overline{f r}$ & 1.5 & & 6.0 & 1.0 & & & 6.0 & 4.6 \\
\hline & $\sigma$ & 0.7 & & 7.1 & 0.0 & & & 3.8 & 4.1 \\
\hline \multirow[t]{4}{*}{ Joda-Time } & $s t$ & 0.0 & 3.0 & 0.0 & 0.0 & 0.0 & 0.0 & 5.0 & 8.0 \\
\hline & $f r$ & 0.0 & 5.0 & 0.0 & 0.0 & 0.0 & 0.0 & 26.0 & 31.0 \\
\hline & $\overline{f r}$ & & 1.7 & & & & & 5.2 & 3.9 \\
\hline & $\sigma$ & & 0.6 & & & & & 1.5 & 2.2 \\
\hline \multirow[t]{4}{*}{ JFreechart } & st & 1.0 & 1.0 & 0.0 & 0.0 & 0.0 & 0.0 & 0.0 & 2.0 \\
\hline & $f r$ & 6.0 & 6.0 & 0.0 & 0.0 & 0.0 & 0.0 & 0.0 & 12.0 \\
\hline & $\overline{f r}$ & 6.0 & 6.0 & & & & & & 6.0 \\
\hline & $\sigma$ & NA & NA & & & & & & 0.0 \\
\hline \multirow[t]{4}{*}{ XWiki } & $s t$ & 20.0 & 4.0 & 0.0 & 6.0 & 1.0 & 0.0 & 20.0 & 51.0 \\
\hline & $f r$ & 535.0 & 39.0 & 0.0 & 131.0 & 8.0 & 0.0 & 687.0 & 1400.0 \\
\hline & $\overline{f r}$ & 26.8 & 9.8 & & 21.8 & 8.0 & & 34.4 & 27.5 \\
\hline & $\sigma$ & 33.3 & 3.7 & & 22.2 & NA & & 47.0 & 37.0 \\
\hline \multirow[t]{4}{*}{ Elasticsearch } & $s t$ & 18.0 & 10.0 & 6.0 & 0.0 & 1.0 & 7.0 & 34.0 & 76.0 \\
\hline & $f r$ & 222.0 & 152.0 & 102.0 & 0.0 & 15.0 & 135.0 & 717.0 & 1343.0 \\
\hline & $\overline{f r}$ & 12.3 & 15.2 & 17.0 & & 15.0 & 19.3 & 21.1 & 17.7 \\
\hline & $\sigma$ & 9.8 & 9.2 & 18.0 & & NA & 11.9 & 13.4 & 12.5 \\
\hline \multirow[t]{4}{*}{ Total } & $s t$ & 49.0 & 24.0 & 14.0 & 10.0 & 9.0 & 7.0 & 87.0 & 200.0 \\
\hline & $f r$ & 782.0 & 212.0 & 135.0 & 144.0 & 34.0 & 135.0 & 1560.0 & 3002.0 \\
\hline & $\overline{f r}$ & 16.0 & 8.8 & 9.6 & 14.4 & 3.8 & 19.3 & 17.9 & 15.0 \\
\hline & $\sigma$ & 23.9 & 8.5 & 13.3 & 19.3 & 4.8 & 11.9 & 26.3 & 22.3 \\
\hline
\end{tabular}

JCrashPack is extensible and publicly available on GitHub. ${ }^{8}$ We provide guidelines to add new crashes to the benchmark and make a pull request to include them in JCrashPack master branch. The detailed numbers for each stack trace and its project are available on the JCrashPack website.

\footnotetext{
${ }^{8}$ At https://github.com/STAMP-project/JCrashPack
} 


\section{Running Experiments with ExRunner}

We combine JCrashPack with ExRunner, a tool that can be used for running experiments with a given stack trace-based crash reproduction tool. This tool (i) facilitates the automatic parallel execution of the crash reproduction instances, (ii) ensures robustness in the presence of failures during the crash reproduction failure, and (iii) allows to plug different crash reproduction tools to allow a comparison of their capabilities.

Figure 2 gives an overview of ExRunner architecture. The job generator takes as input the stack traces to reproduce, the path to the Jar files associated to each stack trace, and the configurations to use for the stack trace reproduction tool under study. For each stack trace, the job generator analyzes the stack frames and discards those with a target method that does not belong to the target system, based on the package name. For instance, frames with a target method belonging to the Java SDK or other external dependencies are discarded from the evaluation. For each configuration and stack trace, the job generator creates a new job description (i.e., a JSON object with all the information needed to run the tool under study) and adds it to a queue.

To speed-up the evaluation, ExRunner multithreads the execution of the jobs. The number of threads is provided by the user in the configuration of ExRunner and depends on the resources available on the machine and required by one job execution. Each thread picks a job from the waiting queue and executes it. ExRunner users may activate an observer that monitors the jobs and takes care of killing (and reporting) those that do not show any sign of activity (by monitoring the job outputs) for a user-defined amount of time. The outputs of every job are written to separate files, with the generated test case (if any) and the results of the job execution (output results from the tool under study).

For instance, when used with EvoCrash, the log files contain data about the target method, progress of the fitness function value during the execution, and branches covered by the execution of the current test case (in order to see if the line where the exception is thrown is reached). In addition, the results contain information about the progress of search (best fitness function, best line coverage, and if the target exception is thrown), and number of fitness evaluations performed by EvoCrash in an output CSV file. If EvoCrash succeeds to replicate the crash, the generated test is stored separately.

As mentioned by Fraser et al. (2013a), any research tool developed to generate test cases may face specific challenges. One of these is long (or infinite) execution time of the test during the generation process. To manage this problem, EvoSuite uses a timeout for each test execution, but sometimes it fails to kill sub-processes spawned during the search (Fraser

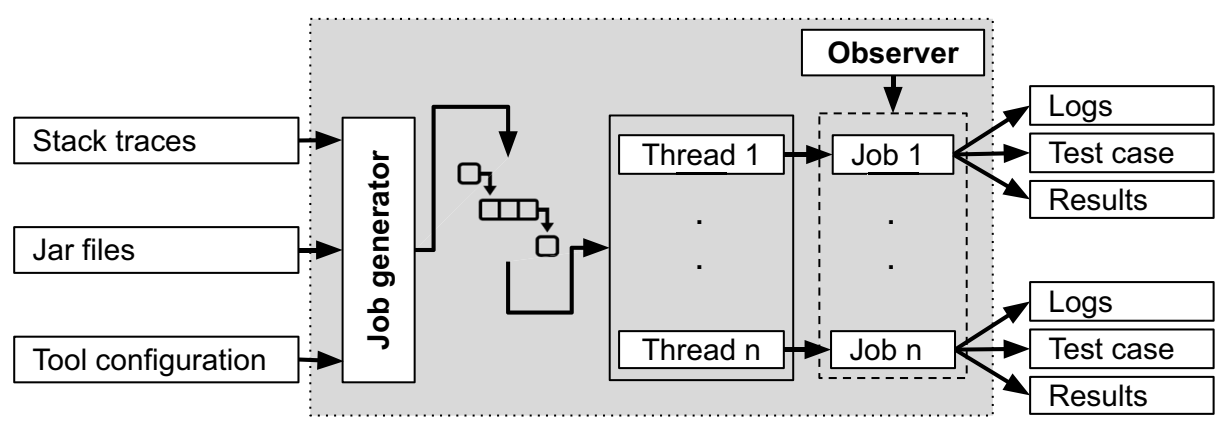

Fig. 2 ExRunner overview 
and Arcuri 2013a). We also experienced EvoCrash freezing during our evaluation. In order to handle this problem, ExRunner creates an observer to check the status of each thread executing an EvoCrash instance. If one EvoCrash execution does not respond for 10 minutes (66\% of the expected execution time), the Python script kills the EvoCrash process and all of its spawned threads.

Another challenge relates to garbage collection: we noticed that, at some point of the execution, one job (i.e., one JVM instance) allocated all the CPU cores for the execution of the garbage collector, preventing other jobs to run normally. Moreover, since EvoCrash allocates a large amount of heap space to each sub-process responsible to generate a new test case (since the execution of the target application may require a large amount of memory) (Fraser and Arcuri 2013a), the garbage collection process could not retrieve enough memory and got stuck, stopping all jobs on the machine. To prevent this behaviour, we set -XX:ParallelGCThreads JVM parameter to 1, enabling only one thread for garbage collection, and limited the number of parallel threads per machine, depending on the maximal amount of allocated memory space. We set the number of active threads to 5 for running on virtual machines, and 25 for running on two powerful machines. Using the logging mechanism in EvoCrash, we are able to see when individual executions ran out of memory.

ExRunner is available together with JCrashPack. ${ }^{9}$ It presently has only been used to perform EvoCrash benchmarking, yet it has been designed to be extensible to other available stack trace reproduction tools using a plugin mechanism. Integrating another crash reproduction tool requires the definition of two handlers, called by ExRunner: one to run the tool with the inputs provided by ExRunner (i.e. the stack trace, the target frame, and the classpath of the software under test); and one to parse the output produced by the tool to pick up relevant data (e.g., the final status of the crash reproduction, progress of the tool during the execution, etc.). Relevant data are stored in a CSV file, readily available for analysis. ${ }^{10}$

\section{Application to EvoCrash: Setup}

Having JCrashPack available allowed us to perform an extensive evaluation of EvoCrash, a state-of-the-art tool in search-based crash replication (Soltani et al. 2018). Naturally, our first research question deals with the capability of EvoCrash to reproduce crashes from JCrashPack:

\section{$\mathbf{R Q}_{1.1} \quad$ To what extent can EvoCrash reproduce crashes from JCrashPack?}

Since the primary goal of our evaluation is to identify current limitations, we refine the previous research question to examine which frames of the different crashes EvoCrash is able to reproduce:

\section{$\mathbf{R Q}_{1.2}$ To what extent can EvoCrash reproduce the different frames of the crashes from JCrashPack?}

The diversity of crashes in JCrashPack also allows us to investigate how certain types of crashes affect reproducibility. Thus, we investigate whether the exception type and the project nature have an influence on the reproduction rate:

\footnotetext{
${ }^{9}$ See https://github.com/STAMP-project/ExRunner.

${ }^{10}$ The ExRunner documentation includes a detailed tutorial describing how to proceed, available at https://github.com/STAMP-project/EvoCrash-JCrashPack-application\#run-other-crash-replication-tools-with -exrunner.
} 
$\mathbf{R Q}_{2.1}$ How does project type influence performance of EvoCrash for crash reproduction?

In addition, different types of projects might have impact on how costly it is to reproduce the reported crashes for them. The second research question studies the influence of the exception and project type on the performance of EvoCrash:

$\mathbf{R Q}_{2.2}$ How does exception type influence performance of EvoCrash for crash reproduction?

Finally, we seek to understand why crashes could not be reproduced:

$\mathbf{R Q}_{3} \quad$ What are the main challenges that impede successful search-based crash reproduction?

\subsection{Evaluation Setup}

Number of Executions Due to the randomness of Guided Genetic Algorithm in EvoCrash, we executed the tool multiple times on each frame. The number of executions has to strike a balance between the threats to external validity (i.e., the number of stack traces considered) and the statistical power (i.e., number of runs) (Fraser and Arcuri 2014b; Arcuri and Briand 2014). In our case, we do not compare EvoCrash to other tools (see for instance Soltani et al. 2017, 2018), but rather seek to identify challenges for crash reproduction. Hence we favor external validity by considering a larger amount of crashes compared to previous studies (Soltani et al. 2018) and ran EvoCrash 10 times on each frame. In total, we executed 18,590 EvoCrash runs.

Search Parameters We used the default parameter values (Arcuri and Fraser 2011; Fraser and Arcuri 2014b) with the following additional configuration options: we chose to keep the reflection mechanisms, used to call private methods, deactivated. The rationale behind this decision is that using reflection can lead to generating invalid objects that break the class invariant (Liskov and Guttag 2000) during the search, which results in test cases helplessly trying to reproduce a given crash (Chen and Kim 2015).

After a few trials, we also decided to activate the implementation of functional mocking available from EvoSuite (Arcuri et al. 2017) in order to minimize possible risks of environmental interactions on crash reproduction. Functional mocking works as follows: when, in a test case, a statement that requires new specific objects to be created (as parameters of a method call for instance) is inserted, either a plain object is instantiated by invoking its constructor, or (with a defined probability, left to its default value in our case) a mock object is created. This mock object is then refined using when-thenReturn statements, based on the methods called during the execution of the generated test case. Functional mocking is particularly useful in the cases where the required object cannot be successfully initialized (for instance, if it relies on environmental interactions or if the constructor is accessible only trough a factory).

Investigating the impact of those parameters and other parameters (e.g., crossover rate, mutation rate, etc. to overcome the challenges as identified in $\mathbf{R} \mathbf{Q}_{3}$ ) is part of our future work.

Search Budget Since our evaluation is executed in parallel on different machines, we choose to express the budget time in terms of number of fitness evaluations: i.e., the number of times the fitness function is called to evaluate a generated test case during the execution of the guided generic algorithm. We set this number to 62,328 , which corresponds to the 
average number of fitness evaluations performed by EvoCrash when running it during 15 minutes on each frame of a subset of 4 randomly selected stack traces on one out of our two machines. Both of the machines have the same configuration: A cluster running Linux Ubuntu 14.04.4 LTS with 20 CPU-cores, 384 GB memory, and a 482 GB hard drive.

We partitioned the evaluation into two, one per available machine: all the stack traces with the same kind of exception have been run on one machine for 10 rounds. For each run, we measure the number of fitness evaluations needed to achieve reproduction (or the exhaustion of the budget if EvoCrash fails to reproduce the crash) and the best fitness value achieved by EvoCrash ( 0 if the crash is reproduced and higher otherwise). The whole process is managed using ExRunner. The evaluation itself was executed during 10 days on our 2 machines.

\section{Application to EvoCrash: Results}

In this section, we answer the first two research questions on the extent to which the selected crashes and their frames were reproduced and the impact of the project and the exception type on the performance of EvoCrash. We detail the results by analyzing the outcome of EvoCrash in a majority of 10 executions for each frame of each stack trace. We classify the outcome of each execution in one of the five following categories:

reproduced: when EvoCrash generated a test that successfully reproduced the stack trace at the given frame level;

ex. thrown: when EvoCrash generated a test that cannot fully reproduce the stack trace, but covers the target line and throws the desired exception. The frames of the exception thrown, however, do not contain all the original frames;

line reached: when EvoCrash generated a test that covers the target line, but does not throw the desired exception;

line not reached: when none of the tests produced by EvoCrash could cover the target line within the available time budget; and

aborted: when EvoCrash could not generate an initial population to start the search process.

Each outcome denotes a particular state of the search process. For the reproduced frames, EvoCrash could generate a crash-reproducing test within the given time budget (here, 62,328 fitness evaluations). For the frames that could not be reproduced, either EvoCrash exhausted the time budget (for ex. thrown, line reached, and line not reached outcomes) or could not perform the guided initialization (i.e., generate at least one test case with the target method) and did not start the search process (aborted outcomes). For instance, if the class in the target frame is abstract, EvoCrash may fail to find an adequate implementation of the abstract class to instantiate an object of this class during the guided initialization.

\subsection{Crash Reproduction Outcomes (RQ1)}

For $\mathbf{R} \mathbf{Q}_{1}$, we first look at the reproduced and non-reproduced crashes to answer $\mathbf{R} \mathbf{Q}_{1.1}$. If EvoCrash was successful in reproducing any frame of a stack trace in a majority of 10 executions, we count the crash as a reproduced crash. Otherwise, we count the crash as not reproduced. To answer $\mathbf{R Q}_{1.2}$, we detail the results by analyzing the outcome of EvoCrash in a majority of 10 executions for each frame of each stack trace. 


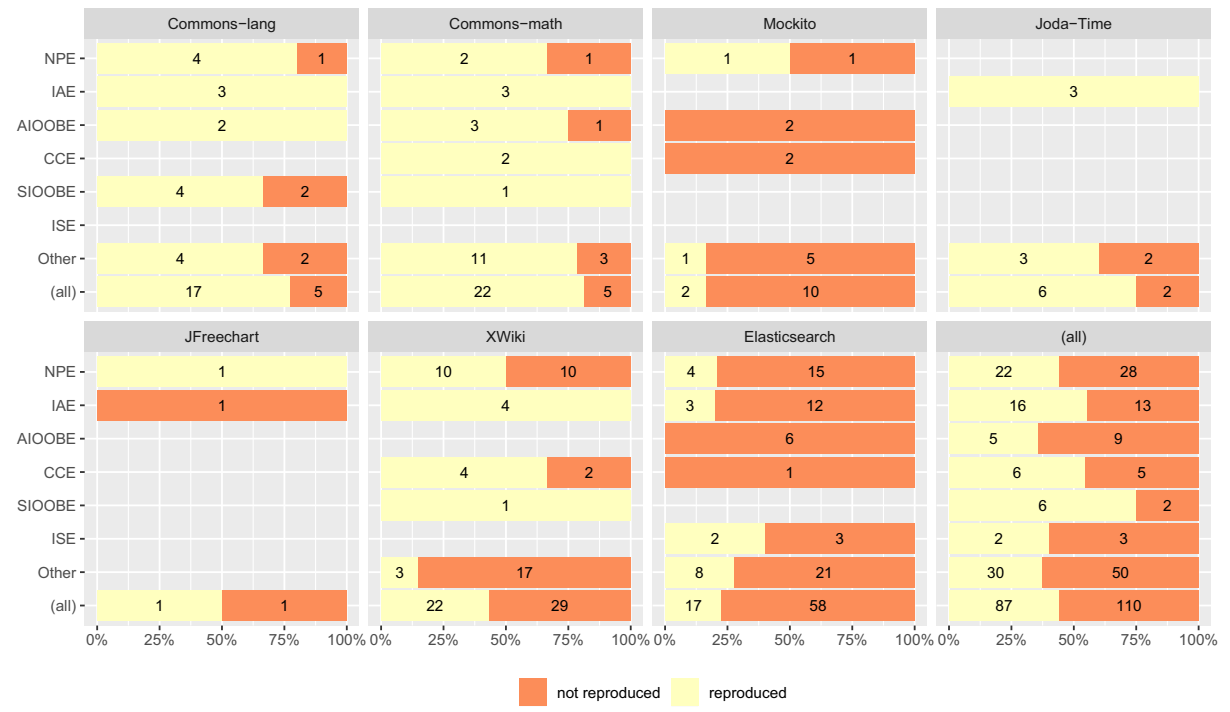

Fig. 3 Reproduction outcome for the different crashes

Figure 3 shows the number of reproduced and not reproduced crashes for each project (and all the projects) and type of exception. EvoCrash is successful in reproducing the majority of crashes (more than 75\%) from Commons-lang, Commons-math, and Joda-Time. For the other projects, EvoCrash reproduced $50 \%$ or less of the crashes, with only 2 out of 12 crashes reproduced for Mockito. Crashes with an IllegalArgumentException are the most frequently reproduced crashed: 16 out of $29(55 \%)$.

Before detailing the results of each frame of each crash, we first look at the frame levels that could be reproduced. Figure 4 presents for the 87 stack traces that could be reproduced, the distribution of the highest frame level that could be reproduced for the different crashes

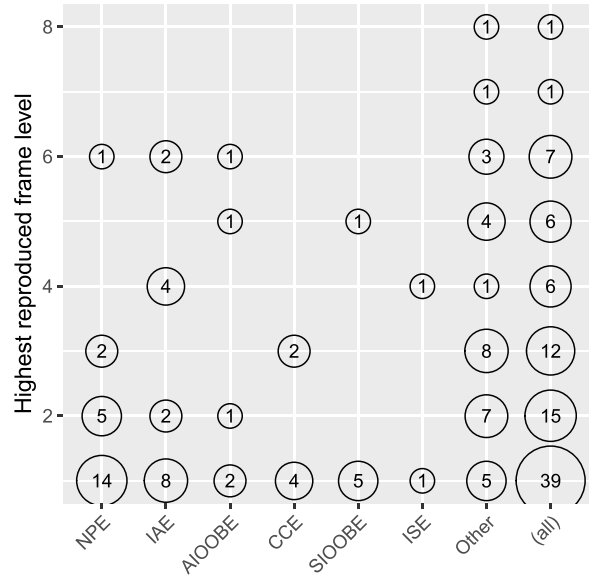

(a) In each type of exception

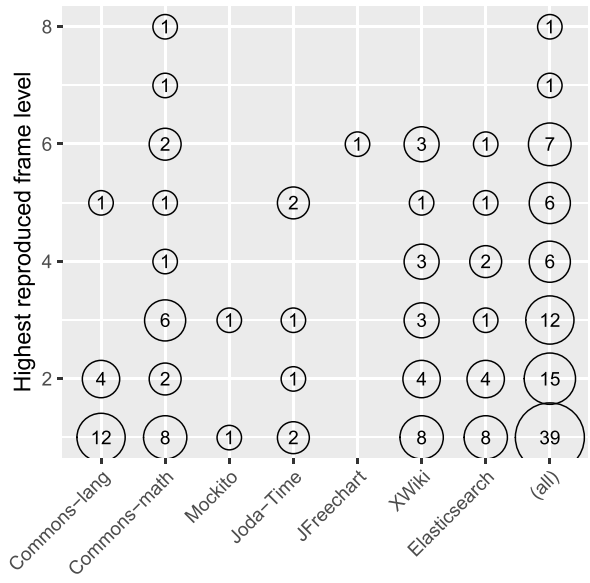

(b) In each type of application

Fig. 4 Highest reproduced frame levels 
for each type of exception (in Fig. 4a) and each application (in Fig. 4b). As we can see, EvoCrash replicates lower frame levels more often than higher levels. For instance, for 39 out of the 87 reproduced stack traces, EvoCrash could not reproduce frames beyond level 1 and could reproduce frames up to level 5 for only 9 crashes.

Figure $4 \mathrm{a}$ indicates that EvoCrash can replicate only the first frame in 14 out of $22 \mathrm{NPE}$ crashes, while there is only one NPE crash for which EvoCrash could reproduce a frame above level 3. In contrast, it is more frequent for EvoCrash to reproduce higher frame levels of IAE stack traces: the highest reproduced frames in 6 out of 16 IAE crashes are higher than 3 . Those results suggest that, when trying to reproduce a crash, propagating an illegal argument value trough a chain of method calls (i.e., the frames of the stack trace) is easier than propagating a null value. According to Fig. 4b, EvoCrash can reproduce frames higher than 6 only for Commons-math crashes. The highest reproduced frames in most of the reproduced crashes in this project are higher than level 2 (12 out of 22). In contrast, for Elasticsearch the highest reproduced frame is 1 in most of the crashes.

Both the number of crashes reproduced and the highest level at which crashes could be reproduced confirm the relevance of our choice to consider crashes from XWiki and Elasticsearch, for which the average number of frames (resp. 27.5 and 17.7) is higher than for Defects4J projects (at most 6.0 for JFreeChart), as they represent an opportunity to evaluate and understand current limitations.

\subsubsection{Frames Reproduction Outcomes}

To answer $\mathbf{R} \mathbf{Q}_{1.2}$, we analyze the results for each frame individually. Figure 5 presents a summary of the results with the number of frames for the different outcomes. Figure 6 details the same results by application and exception.

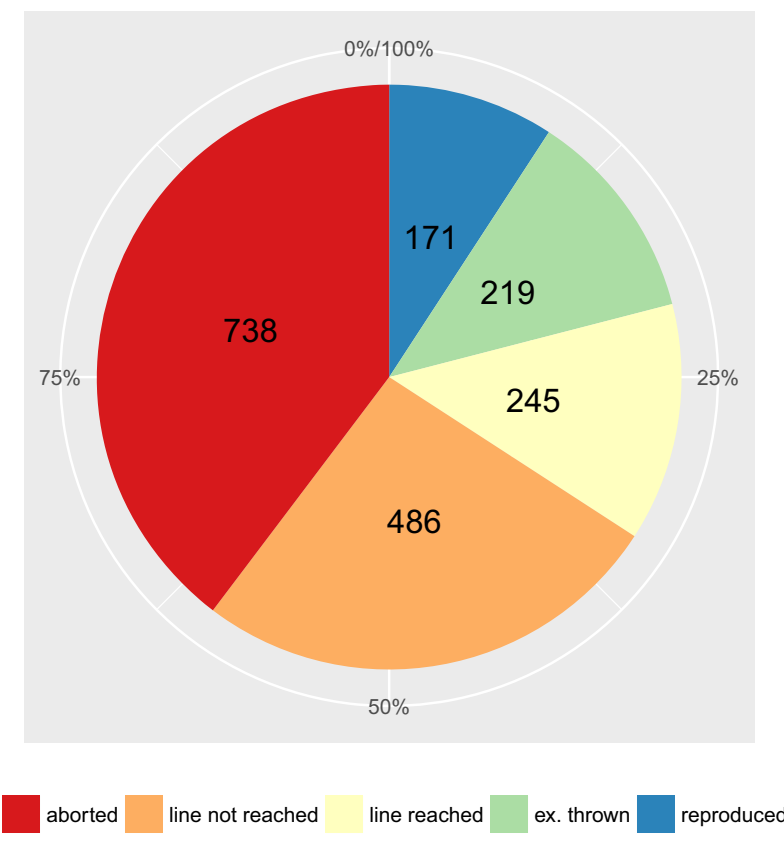

Fig. 5 An overview of the reproduction outcome 


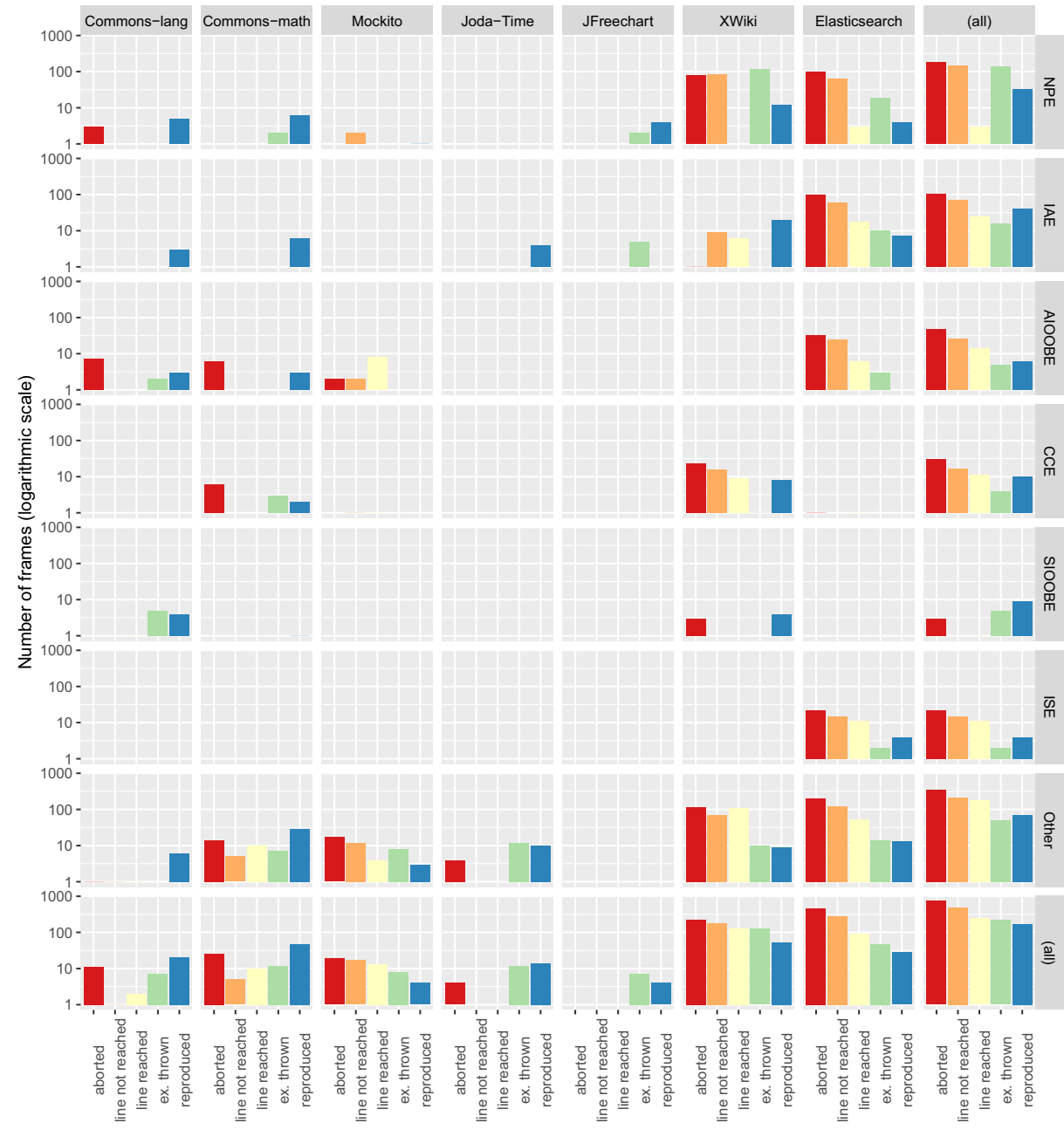

Fig. 6 Detailed reproduction outcome for the different frames

Overall, we see in Fig. 5 that EvoCrash reproduced 171 frames (out of 1,859), from 87 different crashes (out of 200) in the majority of the ten rounds. If we consider the frames for which EvoCrash generated a crash-reproducing test at least once in the ten rounds, the number of reproduced frames increases to 201 (from 96 different crashes). In total, EvoCrash exhausted the time budget for 950 frames: 219 with a test case able to throw the target exception, 245 with a test case able to reach the target line, and 486 without a test case able to reach the line. EvoCrash aborted the search for 738 frames, 455 of which were from Elasticsearch, the application for which EvoCrash had the most difficulties to reproduce a stack trace.

Figure 6 details the results by applications (columns) and exceptions (lines). The last line (resp. column), denoted (all), provides the global results for the applications (resp. exceptions). In the remainder of this section, we discuss the results for the different applications and exceptions. 


\subsubsection{Defects4J Applications}

For the Defects4J applications, presented in the first five columns in Fig. 6, in total, 90 (out of 244) of the frames from 48 (out of 71) different crashes were reproduced. For 94 frames, EvoCrash exhausted the time budget (46 ex. thrown, 25 line reached, and 23 line not reached) and aborted for 60 frames from the Defects4J projects.

In particular, only 4 frames out of 61 frames for Mockito were successfully reproduced. For instance, EvoCrash could not reproduce MOCKITO-4b, which has only one frame. From our evaluation, we observe that one very common problem when trying to reproduce a ClassCastException is to find which class should be used to trigger the exception.

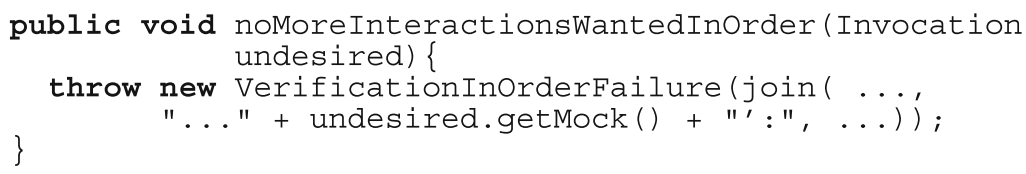

The exception happens when the undesired.getMock () call returns an object that cannot be cast to String. During the search, EvoCrash mocks the undesired object and assigns some random value to return when the getMock method is called. EvoCrash generates a test able to cover the target line, but failing to trigger an exception. Since the signature of this method is Object getMock (), EvoCrash assigns only random Object values to return, where, from the original stack trace, a Boolean value is required to trigger the exception.

\subsubsection{XWiki and Elasticsearch}

XWiki is one of the industrial open source cases in the evaluation, for which 53 (out of 706) frames were successfully reproduced, 430 could not be reproduced with the given time budget (125 ex. thrown, 127 line reached, and 178 line not reached), and 223 aborted during the generation of the initial population. EvoCrash reproduced only 28 (out of 909) frames from Elasticsearch, for which, the majority of frames (455) aborted during the generation of the initial population. However, EvoCrash was able to start the search for 426 frames (48 ex. thrown, 93 line reached, and 285 line not reached).

Variability of the Reproductions We also observed that XWiki and Elasticsearch have the highest variability in their outcomes. For XWiki (resp. Elasticsearch), 4 (resp. 3) frames that could be reproduced in a majority of time could however not be reproduced 10 out of 10 times, compared to 2 frames for Commons-lang and Commons-math. This could indicate a lack of guidance in the current fitness function of EvoCrash. For instance, for the Elasticsearch crash ES-26833, EvoCrash could only reproduce the third frame 4 times out of 10 and was therefore not considered as reproduced. After a manual inspection, we observed that EvoCrash gets stuck after reaching the target line and throwing the expected exception. From the intermediate test cases generated during the search, we see that the exception is not thrown by the target line, but a few lines after. Since the fitness value improved, EvoCrash got stuck into a local optima, hence the lower frequency of reproduction for that frame. ${ }^{11}$ Out future work includes improvement of the guidance in the fitness function and a full investigation of the fitness landscape to decrease the variability of EvoCrash outcomes.

\footnotetext{
${ }^{11}$ A detailed analysis is available at https://github.com/STAMP-project/EvoCrash-JCrashPack-application/ blob/master/results/manual-analysis/Elasticsearch/ES-26833.md
} 
Importance of Large Industrial Applications Compared to Defects4J and XWiki applications, the crash reproduction rate drops from $36.9 \%$ for Defects $4 \mathrm{~J}$, to $7.5 \%$ for XWiki, and only $3 \%$ for Elasticsearch. Those results emphasize the importance of large industrial applications for the assessment of search-based crash reproduction and enforce the need of context-driven software engineering research to identify relevant challenges (Briand et al. 2017).

Additionally to the larger variability of reproduction rate, we observe that frequent use of Java generics and static initialization, and most commonly, automatically generating suitable input data that resembles http requests are among the major reasons for the encountered challenges for reproducing Elasticsearch crashes. In Section 8 we will describe 14 categories of challenges that we identified as the underlying causes for the presented execution outcomes.

\subsubsection{Exceptions}

The lines in Fig. 6 presents the outcomes for the different exceptions. In particular, NPE, IAE, AIOOBE, and CCE are the most represented exceptions in JCrashPack. For those exceptions, EvoCrash could reproduce, respectively, 32 (out of 499), 40 (out of 250), 6 (out of 99), and 10 (out of 72) frames. Looking at the reproduction frequency, IAE is the most frequently reproduced exception (16\%), followed by CCE (13.8\%), NPE (6.4\%), and AIOOBE (6\%).

This contrast with the number of frames for which EvoCrash aborted the search, where NPE has the lowest frequency (181 frames, $36.2 \%$ ), followed by IAE (101 frames, $40.4 \%$ ), CCE (30 frames, 41.6\%), and AIOOBE (48 frames, 48.4\%). Interestingly, those numbers show that EvoCrash is able to complete the guided initialization for NPEs more often than for other exceptions.

Figure 6 also shows that the number of test cases that reach the line is low for NPEs, meaning that whenever EvoCrash generates at test able to cover the line (line reached), the evolution process will be able to progress and generate another test that throws an exception (ex. thrown).

Summary $\left(\mathbf{R Q}_{1}\right)$ To what extent can EvoCrash reproduce crashes from JCrashPack, and how far it can proceed in the stack traces? Overall, EvoCrash reproduced 171 frames (out of $1,859-9 \%$ ), from 87 different crashes (out of $200-43.5 \%$ ) in a majority out of 10 executions. Those numbers climb to 201 frames (10.8\%) from 96 crashes $(48 \%)$ if we consider at least one reproduction in one of the 10 executions. In most of the reproduced crashes, EvoCrash can only reproduce the first two frames. It indicates that since EvoCrash needs higher accuracy in setting the state of the software under test for reproducing higher frames, increasing the length of the stack trace reduces the chance of this tool for crash reproduction. When looking at larger industrial applications, the crash reproduction rates drop from $36.9 \%$ for Defects4J to $7.5 \%$ for XWiki and 3\% for Elasticsearch. The most frequently reproduced exceptions are IllegalArgumentExceptions. The exceptions for which EvoCrash is the most frequently able to complete the guided initialization are NullPointerExceptions.

\subsection{Impact of Exception Type and Project on Performance (RQ2)}

To identify the distribution of fitness evaluations per exception type and project, we filtered the reproduced frames out of the 10 rounds of execution. Tables 5 and 6 present the statistics for these executions, grouped by application and exception types, respectively. 
Table 5 Statistics for the average number of fitness evaluations for the reproduced frames (fr) belonging to different stack traces (st), grouped by applications, out of 10 rounds of execution. The confidence Interval (CI) is calculated for the median bootstrapping with 100,000 runs, at a 95\% confidence level

\begin{tabular}{lllllllll}
\hline Applications & st & fr & Min & Lower Quart. & Median CI & Med. & Upper Quart. & Max \\
Com.-lang & 19 & 213 & 1 & 2.0 & {$[5.0,22.0]$} & 15.0 & 237.0 & 52,240 \\
Com.-math & 24 & 471 & 1 & 13.0 & {$[124.0,211.0]$} & 178.0 & $1,046.5$ & 58,731 \\
Mockito & 2 & 40 & 1 & 1.0 & {$[1.0,1.0]$} & 1.0 & 5.2 & 138 \\
Joda-Time & 6 & 138 & 1 & 15.5 & {$[79.1,369.0]$} & 253.5 & $1,290.2$ & 40,189 \\
JFreechart & 1 & 41 & 1 & 10.0 & {$[-292.0,350.0]$} & 221.0 & $1,188.0$ & 20,970 \\
XWiki & 25 & 531 & 1 & 2.5 & {$[14.0,30.0]$} & 23.0 & 209.0 & 34,089 \\
Elasticsearch & 19 & 287 & 1 & 4.0 & {$[5.0,32.0]$} & 23.0 & 125.0 & 17,461 \\
Total & 96 & 1721 & 1 & 4.0 & {$[34.0,59.0]$} & 48.0 & 534.0 & 58,731 \\
\hline
\end{tabular}

We filtered out the frames that were not reproduced to analyze the impact of project and exception types on the average number of fitness evaluations and, following recommendations by Arcuri and Briand (2014), we replaced the test of statistical difference by a confidence interval. For both groups, we calculated confidence intervals with a 95\% confidence level for medians with bootstrapping with 100,000 runs. ${ }^{12}$

As Table 5 shows, for four projects (Commons-lang, Mockito, XWiki, and Elasticsearch) the median number of fitness evaluations is low. On the contrary, the cost of crash reproductions for Commons-math, Joda-Time, and JFreechart are higher in comparison to the rest of projects. By comparing those results with the projects sizes reported in Table 3, where the largest projects are XWiki (with $\overline{N C S S}=177.84 k$ ) and Elasticsearch (with $\overline{N C S S}=124.36 k$ ), we observe that the effort required to reproduce a crash cannot be solely predicted by the project size. This is consistent with the intuition that the difficulty of reproducing a crash only depends on the methods involved in the stack trace.

Similarly, according to Fig. 1a, the average CCN for Mockito, XWiki, and Elasticsearch is lower compared to other projects. Table 5 shows that reproducing crashes from these projects is less expensive, and that reproducing crashes from Commons-math, Joda-Time, and JFreechart, which all have higher average $\mathrm{CCN}$, is more expensive. We also observe that the average $\mathrm{CCN}$ for Commons-lang is high, however, contradicting the intuition that crashes from projects higher $\mathrm{CCN}$ are more expensive to reproduce, the cost for reproducing crashes in Commons-lang is low compared to other projects. This can be explained by the levels of the frames reproduced by EvoCrash: according to Fig. 4, the average level of the reproduced frames in the crashes from Commons-lang is low compared to the other projects and, as we discussed in the previous section, reproducing crashes with fewer frames is easier for EvoCrash.

In general, we observe that the performance of EvoCrash depends on the complexity of the project and the frame level in the stack trace. Future work includes further investigations to determine which other factors (e.g., code quality) can influence EvoCrash performance.

From Table 6, we observe that for $C C E$, SIOOBE, and $A I O O B E$, the cost of generating a crash-reproducing test case is high, while for $N P E, I A E$, and $I S E$, the cost is lower. One possible explanation could be that generating input data which is in a suitable state for

\footnotetext{
${ }^{12}$ We used the boot function from the boot library in $\mathrm{R}$ to compute the basic intervals with bootstrapping. See https://github.com/STAMP-project/EvoCrash-JCrashPack-application/tree/master/results to reproduce the statistical analysis.
} 
Table 6 Statistics for the average number of fitness evaluations for the reproduced frames ( $\mathbf{f r})$ belonging to different stack traces (st), grouped by exceptions, out of 10 rounds of execution. Confidence Interval (CI) is calculated for median with bootstrapping with 100,000 runs, at $95 \%$ confidence level

\begin{tabular}{lllllllll}
\hline Applications & st & fr & Min & Lower Quart. & Median CI & Med. & Upper Quart. & Max \\
\hline NPE & 26 & 330 & 1 & 6.0 & {$[9.0,63.0]$} & 44.5 & 220.0 & 34,089 \\
IAE & 16 & 399 & 1 & 2.0 & {$[7.0,12.0]$} & 10.0 & 49.0 & 38,907 \\
AIOOBE & 5 & 58 & 1 & 15.5 & {$[252.0,1,104.5]$} & 675.0 & $1,671.2$ & 53,644 \\
CCE & 6 & 103 & 1 & 6.5 & {$[74.0,210.0]$} & 120.0 & 560.0 & 10,197 \\
SIOOBE & 8 & 95 & 1 & 12.5 & {$[122.0,945.0]$} & 505.0 & $2,326.0$ & 52,240 \\
ISE & 2 & 42 & 1 & 1.0 & {$[1.0,3.0]$} & 2.0 & 105.8 & 1,138 \\
Other & 33 & 694 & 1 & 7.0 & {$[99.0,139.0]$} & 125.5 & 825.0 & 58,731 \\
Total & 96 & 1721 & 1 & 4.0 & {$[34.0,59.0]$} & 48.0 & 534.0 & 58,731 \\
\hline
\end{tabular}

causing cast conflicts, or an array which is in the right state to be accessed by an illegal index is often non-trivial.

In contrast, to trigger an NPE, it is often enough to return a null value not checked by the crashing method. For example, Listing 1 shows the stack trace of CHART$4 \mathrm{~b}$, a crash from the JFreeChart application. The crash happens at line 1490 of the createScatterPlot method presented in Listing 2. Listing 3 shows the test case generated by EvoCrash that reproduces the 6th frame (line 6 in Listing 1) of the stack trace. First, the test initializes the mocks used as mandatory parameters values (from line 2 to 4 ), before calling the createScatterPlot method (at line 5). The ds XYDataset mock is used along the various calls (from line 6 to 1 in Listing 1), up to the method getDataRange presented in Listing 4 that triggers the NPE at line 4493. In our case, the null value is returned by the getRendererForDataset call with the propagated ds mock at line 4491.

Considering the presented results in Fig. 6 and Table 5, crash replication for various exceptions may be dependent on project type. Figure 7 presents the results of crash reproduction grouped both by applications and exception types. As the figure shows, the cost of reproducing NPE is lower for Elasticsearch, compared to XWiki and JFreechart, and the cost of reproducing IAE is lower for Commons-lang than for Elasticsearch. We also observe differences in terms of costs of reproducing AIOOBE and SIOOBE for different projects.

Summary $\left(\mathrm{RQ}_{2.1}\right)$ How does project type influence performance of EvoCrash for crash reproduction? We observed that the factors are (i) the complexity of the the project, and (ii)

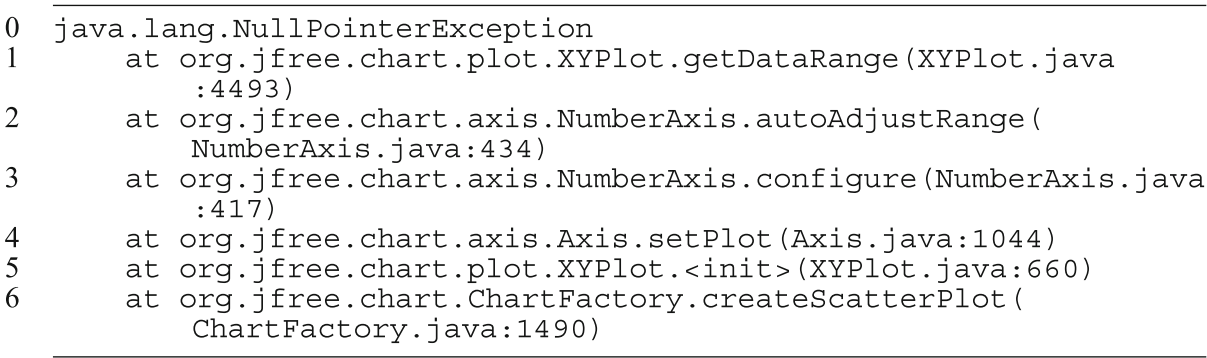

Listing 1 Stack trace for the crash CHART-4b 


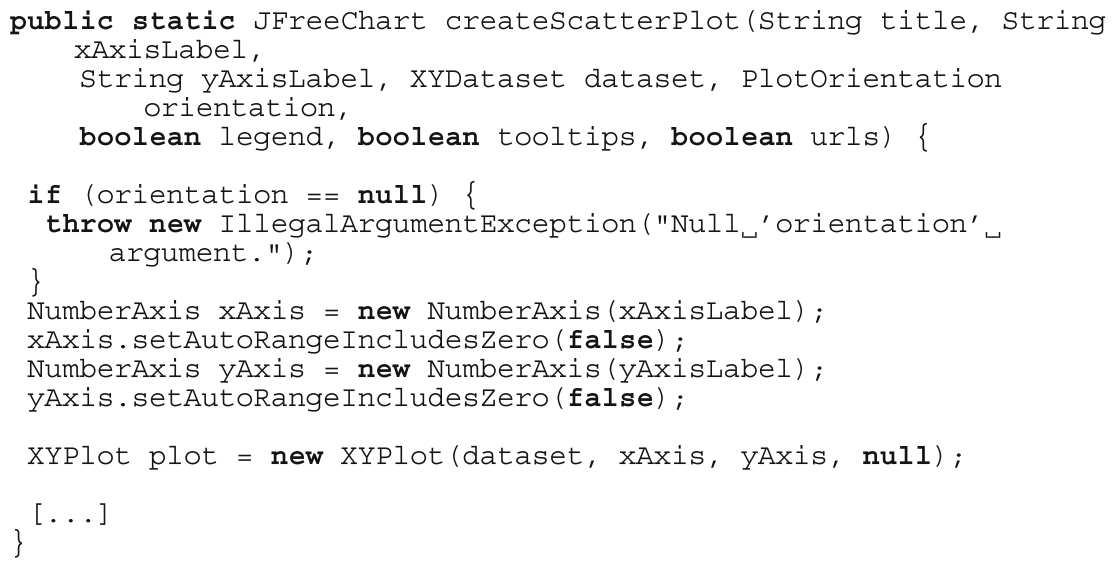

Listing 2 Code excerpt from JFreeChart ChartFactory.java

the level of the reproduced frames (reproducing higher frame requires more effort). Furthermore, we see no link between the size of the project and the effort required to reproduce one of its crashes.

Summary $\left(\mathbf{R Q}_{2.2}\right)$ How does exception type influence performance of EvoCrash for crash reproduction? For the exceptions, we observe that for ClassCastException, ArrayIndexOutOfBoundsException and StringIndexOutOfBoundsException, the cost of generating a crash-reproducing test case is high, while for NullPointerException, IllegalArgumentException, and IllegalStateException, the cost is lower. This result indicates that the cost of reproducing types of exceptions for a non-trivial scenario (e.g., class conflicts or accessing an illegal state of an array) needs a more complex input generation. Furthermore, accessing the corresponding complex state is more time consuming for the search process.

\section{Challenges for Crash Reproduction (RQ3)}

To identify open problems and future research directions, we manually analyzed the execution logs of 1,653 frames that could not be reproduced in any of the 10 executions. This

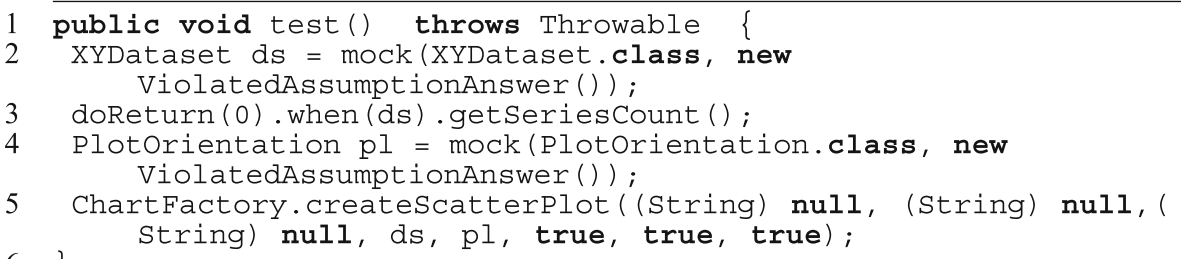

Listing 3 The test case generated by EvoCrash for reproducing the 6th frame of CHART-4b 


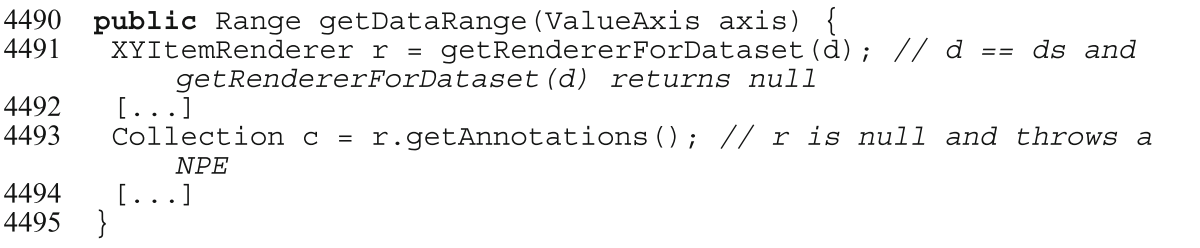

Listing 4 Code excerpt from JFreeChart XYPlot. java

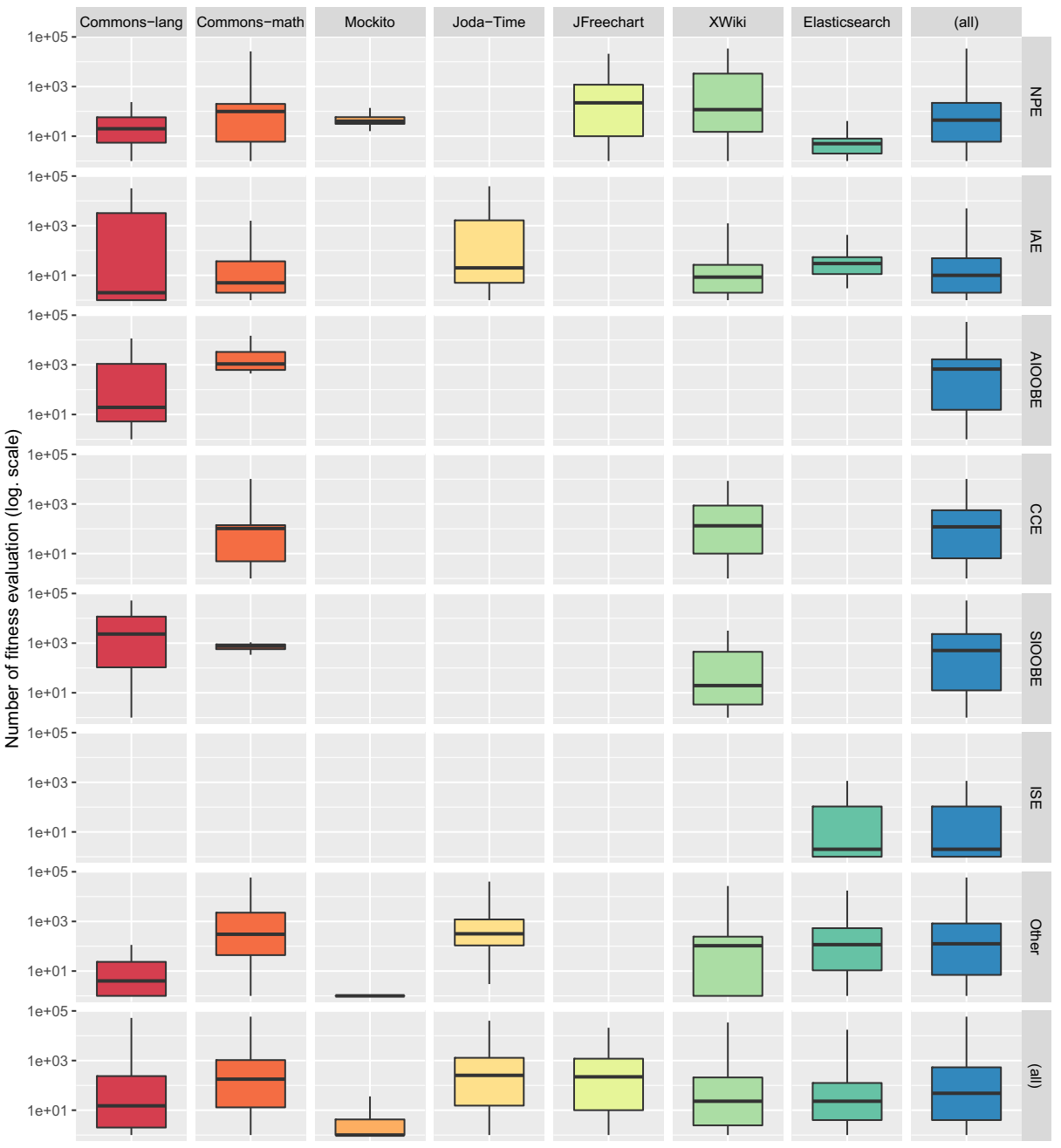

Fig. 7 Average number of fitness evaluations for the reproduced frames for each applications and exception type 
Table 7 Challenges with the number and percentage of frames identified for this challenge

\begin{tabular}{lll}
\hline Category & Frames & Frequency \\
\hline Input Data Generation & 825 & $49.91 \%$ \\
Abstract Class & 242 & $14.64 \%$ \\
Anonymous Class & 142 & $8.59 \%$ \\
Static Initialization & 141 & $8.53 \%$ \\
Complex Code & 118 & $7.14 \%$ \\
Private Inner Class & 56 & $3.39 \%$ \\
Environmental Dependencies & 52 & $3.15 \%$ \\
Irrelevant Frame & 37 & $2.24 \%$ \\
Unknown Sources & 16 & $0.97 \%$ \\
Nested calls & 10 & $0.60 \%$ \\
try/catch & 7 & $0.42 \%$ \\
Interface & 6 & $0.36 \%$ \\
Empty Enum Type & 1 & $0.06 \%$ \\
Total & 1653 & $100 \%$ \\
\hline
\end{tabular}

analysis includes a description of the reason why a frame could not be reproduced. ${ }^{13}$ Based on those descriptions, we grouped the reason of the different failures into 13 categories and identified future research directions. Table 7 provides the number and frequency of frames classified in each category. ${ }^{14}$ The complete categorization table is available in our replication package. ${ }^{15}$

For each challenge, we discuss to what extent it is crash-reproduction-specific and its relation to search-based software testing in general. In particular, for challenges previously identified by the related literature in search-based test case generation, we highlight the differences originating from the crash reproduction context.

\subsection{Input Data Generation}

Generating complex input objects is a challenge faced by many automated test generation approaches, including search-based software testing and symbolic execution (Braione et al. 2017). Usually, the input space of each input is large and generating proper data enabling the search process to cover its goals is difficult.

As we can see from Table 7, this challenge is substantial in search-based crash reproduction. Trying to replicate a crash for a target frame requires to set the input arguments of the target method and all the other calls in the sequence properly such that when calling the target method, the crash happens. Since the input space of a method is usually large, this can be challenging. EvoCrash uses randomly generated input arguments and mock objects as inputs for the target method. As we described in Section 7, we observe that a widespread

\footnotetext{
${ }^{13}$ Available at https://github.com/STAMP-project/EvoCrash-JCrashPack-application/tree/master/results/ manual-analysis.

${ }^{14}$ For each category, we provide illustrative examples from https://github.com/STAMP-project/ EvoCrash-JCrashPack-application/tree/master/results/examples.

${ }^{15}$ The full table is available at https://github.com/STAMP-project/EvoCrash-JCrashPack-application/blob/ master/results/manual-analysis/categorisation.csv.
} 


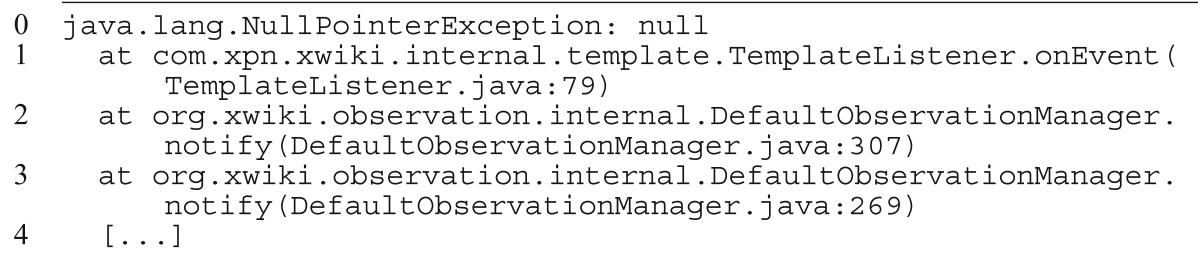

Listing 5 Excerpt of the stack trace for the crash XWIKI-13708

problem when reproducing a ClassCastException (CCE) is to identify which types to use as input parameters such that a CCE is thrown. In the case of a CCE, this information can be obtained from the error message of the exception. Our future work includes harvesting additional information, like error messages, to help the search process.

We also noticed that some stack traces involving Java generic types make EvoCrash abort the search after failing to inject the target method in every generated test during the guided initialization phase. Generating generic type parameters is also a recognized challenge for automated testing tools for Java (Fraser and Arcuri 2014a). To handle these parameters, EvoCrash, based on EvoSuite's implementation (Fraser and Arcuri 2014a), collects candidate types from castclass and instanceof operators in Java bytecode, and randomly assign them to the type parameter. Since the candidate types may themselves have generic type parameters, a threshold is used to avoid large recursive calls to generic types. One possible explanation for the crashes in these cases could be that the threshold is not correctly tuned for the kind of classes involved in the recruited projects. Thus, the tool fails to set up the target method to inject to the tests. Based on the results of our evaluation, handling Java generics in EvoCrash needs further investigation to identify the root cause(s) of the crashes and devise effective strategies to address them.

For instance, EvoCrash cannot reproduce the first frame of crash XWIKI-13708 ${ }^{16}$, presented in Listing 5. The target method onEvent (detailed in Listing 6) has three parameters. EvoCrash could not reach the target line (line 78 in Listing 6) as it failed to generate a fitted value for the second parameter (source). This (Object) parameter should be castable to XWikiDocument and should return values for getXObject () or getAttachment () (using mocking for instance).

Chosen Examples XWIKI-13708, frame 1; ES-22922, frame 5; ES-20479, frame $10 .{ }^{17}$

\subsection{Complex Code}

Generating tests for complex methods is hard for any search-based software testing tool (Harman et al. 2004). In this study, we indicate a method as complex if (i) it contains more than 100 lines of code and high cyclomatic complexity; (ii) it holds nested predicates (Malburg and Fraser 2011; Harman et al. 2004); or (iii) it has the flag problem (Malburg and Fraser 2011; McMinn 2011), which include (at least one) branch predicate with a binary (boolean) value, making the landscape of the fitness function flat and turning the search into a random search (Harman et al. 2004).

\footnotetext{
${ }_{16}$ https://jira.Xwiki.org/browse/XWIKI-13708

${ }^{17}$ See https://github.com/STAMP-project/EvoCrash-JCrashPack-application/blob/master/results/examples/ InputDataGeneration.md.
} 


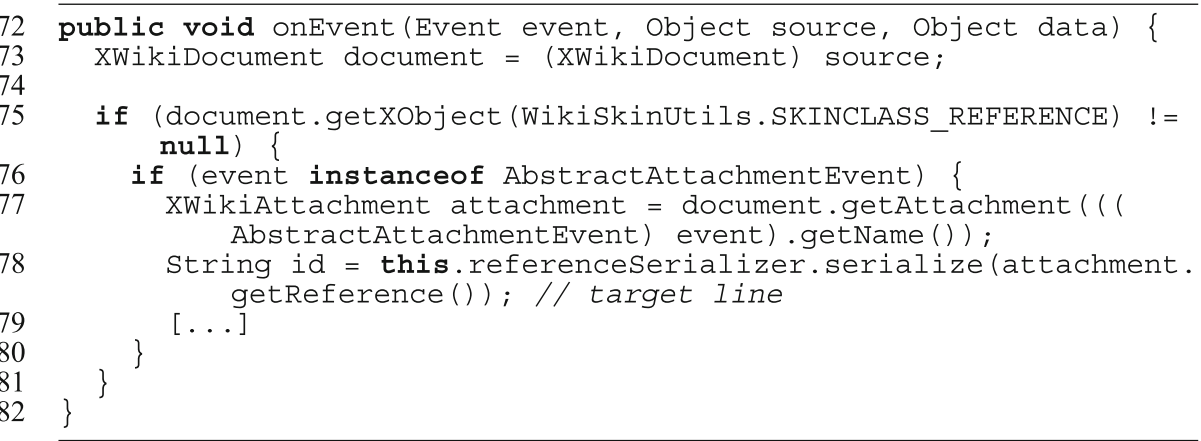

Listing 6 Code excerpt from method onEvent in TemplateListener.java

As presented in Section 2, the first component of the fitness function that is used in EvoCrash encodes how close the algorithm is to reach the line where the exception is thrown. Therefore, frames of a given stack trace pointing to methods with a high code complexity ${ }^{18}$ are more costly to reproduce, since reaching the target line is more difficult.

Handling complex methods in search-based crash reproduction is harder than in general search-based testing. The search process in crash reproduction should cover (in most cases) only one specific path in the software under test to achieve the reproduction. If there is a complex method on this path, the search process cannot achieve reproduction without covering it. Unlike the more general coverage driven search-based testing approach (with line coverage for instance), where the are usually multiple possible executions paths to cover a goal.

Chosen Examples XWIKI-13096, frame 3; ES-22373, frame $10 .^{19}$

\subsection{Environmental Dependencies}

As discussed by Arcuri et al. (2014), generating unit tests for classes which interact with the environment leads to (i) difficulty in covering certain branches which depend on the state of the environment, and (ii) generating flaky tests (Luo et al. 2014), which may sometimes pass, and sometimes fail, depending on the state of the environment. Despite the numerous advances made by the search-based testing community in handling environmental dependencies (Arcuri et al. 2014; Fraser and Arcuri 2014b), we noticed that having such dependencies in the target class hampers the search process. Since EvoCrash builds on top of EvoSuite (Fraser and Arcuri 2013b), which is a search-based unit test generation tool, we face the same problem in the crash reproduction problem as well.

For instance, Listing 7 shows the stack trace of the crash XWIKI-12584. ${ }^{20}$ During the evaluation, EvoCrash could not reproduce any of the frames of this stack trace. During

\footnotetext{
${ }^{18}$ In some cases for Elasticsearch, the failing methods have nearly 300 lines of source code.

${ }^{19} \mathrm{See}$ https://github.com/STAMP-project/EvoCrash-JCrashPack-application/blob/master/results/examples/ ComplexCode.md.

${ }^{20}$ Reported at https://jira.xwiki.org/browse/XWIKI-12584 and analyzed at https://github.com/ STAMP-project/EvoCrash-JCrashPack-application/blob/master/results/manual-analysis/Xwiki/ XWIKI- 12584.md.
} 


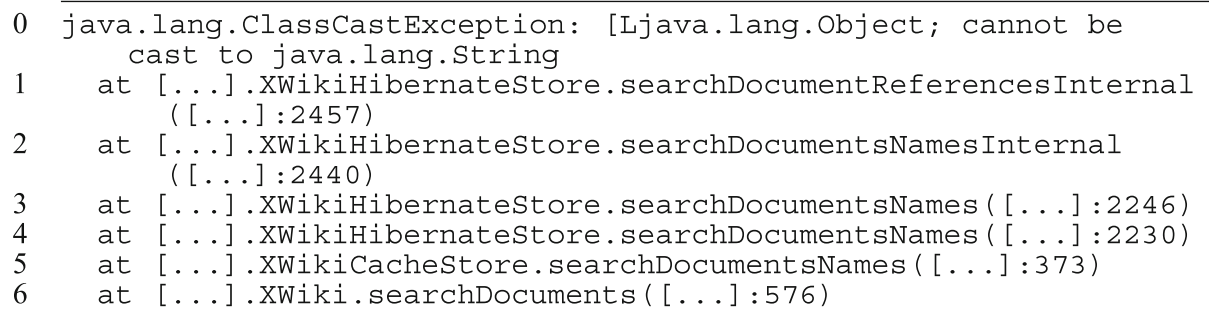

Listing 7 Stack trace for the crash XWIKI-12584

our manual analysis, we discovered that, for the four first frames, EvoCrash was unable to instantiate an object of class XWikiHibernatestore, ${ }^{21}$ resulting in an abortion of the search. Since the class XWikiHibernateStore relies on a connection to an environmental dependency (here, a database), generating unit test requires substantial mocking $\operatorname{code}^{22}$ that is hard to generate for EvoCrash. As for input data generation, our future work includes harvesting and leveraging additional information from existing tests to identify and use relevant mocking strategies.

Chosen Examples ES-21061, frame 4; XWIKI-12584, frame $4 .^{23}$

\subsection{Static Initialization}

In Java, static initializers are invoked only once when the class containing them is loaded. As explained by Fraser and Arcuri (2014b), these blocks may depend on static fields from other classes on the classpath that have not been initialized yet, and cause exceptions such as NullPointerException to be thrown. In addition, they may involve environmental dependencies that are restricted by the security manager, which may also lead to unchecked exceptions being generated.

In our crash reproduction benchmark, we see that about 9\% (see Table 7) of the cases cannot be reproduced as they point to classes that have static initializers. When such frames are used for crash reproduction with EvoCrash, the tool currently aborts the search without generating any crash reproducing test. As Fraser and Arcuri (2014b) discuss, automatically determining and solving all possible kinds of dependencies in static initializers is a nontrivial task that warrants dedicated research.

Chosen Examples ES-20045, frames 1 and 2. ${ }^{24}$

\footnotetext{
${ }^{21}$ See https://github.com/xwiki/xwiki-platform/blob/xwiki-platform-7.2-milestone-2/xwiki-platform-core/ xwiki-platform-oldcore/src/main/java/com/xpn/xwiki/store/XWikiHibernateStore.java

${ }^{22}$ See https://github.com/xwiki/xwiki-platform/blob/xwiki-platform-7.2-milestone-2/xwiki-platform-core/ xwiki-platform-oldcore/src/test/java/com/xpn/xwiki/store/XWikiHibernateStoreTest.java

${ }^{23}$ See https://github.com/STAMP-project/EvoCrash-JCrashPack-application/blob/master/results/examples/ EnvironmentalDependencies.md.

${ }^{24}$ See https://github.com/STAMP-project/EvoCrash-JCrashPack-application/blob/master/results/examples/ StaticInitialisation.md.
} 


\subsection{Abstract Classes and Methods}

In Java, abstract classes cannot be instantiated. Although generating coverage driven unit tests for abstract classes is possible (one would most likely generate unit tests for concrete classes extending the abstract one or use a parametized test to check that all implementations respect the contract defined by the abstract class), when a class under test is abstract, EvoSuite (as the general test generation tool for java) looks for classes on the classpath that extend the abstract class to create object instances of that class. In order to cover (e.g., using line coverage) specific parts of the abstract class, EvoSuite needs to instantiate the right concrete class allowing to execute the different lines of the abstract class.

For crash reproduction, as we can see from Table 7, it is not uncommon to see abstract classes and methods in a stack trace. In several cases from Elasticsearch, the majority of the frames from a given stack trace point to an abstract class. Similarly to coverage-driven unit test generation, EvoCrash needs to instantiate the right concrete class: if EvoCrash picks the same class that has generated the stack trace in the first place, then it can generate a test for that class that reproduces the stack trace. However, if EvoCrash picks a different class, it could still generate a test case that satisfies the first two conditions of the fitness function (section 2). In this last case, the stack trace generated by the test would match the frames of the original stack trace, as the class names and line numbers would differ. The fitness function would yield a value between 0 and 1, but it may never be equal to 0 .

Chosen Examples ES-22119, frames 3 and 4; XRENDERING-422, frame $6 .^{25}$

\subsection{Anonymous Classes}

As discussed in the study by Fraser and Arcuri (2013b), generating automated tests for covering anonymous classes is more laborious because they are not directly accessible. We observed the same challenge during the manual analysis of crash reproduction results generated by EvoCrash. When the target frame from a given crash stack trace points to an anonymous object or a lambda expression, guided initialization in EvoCrash fails, and EvoCrash aborts the search without generating any test.

Chosen Examples ES-21457, frame 8; XWIKI-12855, frames 30 and $31 .^{26}$

\subsection{Private Inner Classes}

Since it is not possible to access a private inner class, and therefore, not possible to directly instantiate it, it is difficult for any test generation tool in Java to create an object of this class. As for anonymous classes, this challenge is also present for crash reproduction approaches. In some crashes, the target frame points to a failing method inside a private inner class. Therefore, it is not possible to directly inject the failing method from this class during the guided initialization phase, and EvoCrash aborts the search.

\footnotetext{
${ }^{25}$ See https://github.com/STAMP-project/EvoCrash-JCrashPack-application/blob/master/results/examples/ AbstractClass.md.

${ }^{26}$ See https://github.com/STAMP-project/EvoCrash-JCrashPack-application/blob/master/results/examples/ AnonymousClass.md.
} 
Chosen Example MATH-58b, frame $3 .^{27}$

\subsection{Interfaces}

In 6 cases, the target frame points to an interface. In Java, similar to abstract classes, interfaces may not be directly instantiated. In these cases also, EvoCrash randomly selects the classes on the classpath that implement the interface and, depending on the class picked by EvoCrash, the fitness function may not reach 0.0 during the search if the class is different from the one used when the input stack trace has been generated. This category is a special case of Abstract classes and methods (described in Section 8.5), however, since the definition of a default behavior for an interface is a feature introduced by Java 8 (Oracle 2019) that has, to the best of our knowledge, not been previously discussed for search-based testing, we choose to keep it as a separate category.

Chosen Example ES-21457, frame 9. ${ }^{28}$

\subsection{Nested Private Calls}

In multiple cases, the target frame points to a private method. As we mentioned in Section 6, those private methods are not directly accessible by EvoCrash. To reach them, EvoCrash detects other public or protected methods which invoke the target method directly or indirectly and randomly choose during the search. If the chain of method calls, from the public caller to the target method, is too long, the likelihood that EvoCrash may fail to pick the right method during the search increases.

In general, calling private methods is challenging for any automated test generation approach. For instance, Arcuri et al. (2017) address this problem by using the Java reflection mechanism to access private methods and private attributes during the search. As mentioned in Section 6.1, this can generate invalid objects (with respect to their class invariants) and lead to generating test cases helplessly trying to reproduce a given crash (Chen and Kim 2015).

Chosen Examples XRENDERING-422, frames 7 to $9 .^{29}$

\subsection{Empty enum Type}

In the stack trace of the ES-25849 crash $^{30}$ the 4 th frame points to an empty enumeration Java type. ${ }^{31}$ Since there are no values in the enumeration, EvoCrash was not able to

\footnotetext{
${ }^{27}$ See https://github.com/STAMP-project/EvoCrash-JCrashPack-application/blob/master/results/examples/ PrivateInnerClass.md.

${ }^{28}$ See https://github.com/STAMP-project/EvoCrash-JCrashPack-application/blob/master/results/examples/ Interface.md.

${ }^{29}$ See https://github.com/STAMP-project/EvoCrash-JCrashPack-application/blob/master/results/examples/ NestedPrivateCalls.md.

${ }^{30} \mathrm{The}$ analysis is available at https://github.com/STAMP-project/EvoCrash-JCrashPack-application/blob/ master/results/manual-analysis/Elasticsearch/ES-25849.md.

${ }^{31}$ See https://github.com/jimczi/elasticsearch/blob/0a4b38b60c2752cdc6de819f5bf3414bd01f88c5/core/src/ main/java/org/elasticsearch/index/fielddata/ordinals/GlobalOrdinalsBuilder.java.
} 
instantiate a value and aborted during the initialization of the population. Frames pointing to code in an empty enumeration Java type should not be selected as target frames and could be filtered out using a preliminary static analysis.

Chosen Example ES-25849, frame 4.

\subsection{Frames with trylcatch}

Some frames have a line number that designates a call inside a try/catch block. When the exception is caught, it is no longer thrown at the specific line given in the trace, rather it is typically handled inside the associated catch blocks. From what we observed, often catch blocks either (i) re-throw a checked exception, which yield chained stack traces with information that is not exactly as the input stack trace but can still be used for crash reproduction; or (ii) $\log$ the caught exception. Since EvoCrash only considers uncaught exceptions that are generated as the result of running the generated test cases during the search, the logged stack traces is presently no use for crash reproduction. Also, even if a stack trace is recorded to an error log, this stack trace is not the manifestation of a crash per se. Indeed, once the exception logged, the execution of the program continues normally.

For instance, for the crash ES-20298, ${ }^{32}$ EvoCrash cannot reproduce the fourth frame of the crash. This frame points to the following method call in a try and catch:

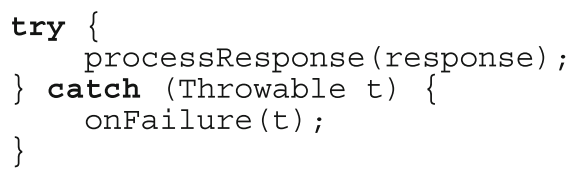

Even if an exception is thrown by the processResponse method, this exception is caught and logged, and the execution of the program continues normally.

Generally, if an exception is caught in one frame, it cannot be reproduced (as it cannot be observed) from higher level frames. For instance, for ES-20298, all frames above level 4 cannot be reproduced since the exception is catch in frame 4 and not propagated to the higher frames. This property of a crash stack trace implies that, for now, depending on where in the trace such frames exist, only a fraction of the input stack traces can actually be used for automated crash reproduction. Future development of EvoCrash can alleviate this limitation by, additionally to the monitoring of uncaught exceptions, read the error log to affecting the propagation of exceptions during execution. However, unlike other branching instructions relying on boolean values, for which classical coverage driven unit test generation can use the branch distance (see Section 2.2.1) to guide the search (McMinn 2004), there is little guidance offered for try/catch instructions since the branching condition is implicit in one or more instructions in the try.

Chosen Example ES-14457, frame 4. ${ }^{33}$

\footnotetext{
${ }^{32}$ Reported at https://github.com/elastic/elasticsearch/issues/20298 and analyzed at https://github.com/ STAMP-project/EvoCrash-JCrashPack-application/blob/master/results/manual-analysis/Elasticsearch/ ES-20298.md

${ }^{33}$ See https://github.com/STAMP-project/EvoCrash-JCrashPack-application/blob/master/results/examples/ TryCatch.md.
} 
1 at org.apache.xerces.parsers.XMLParser.parse (Unknown Source)

2 at org.apache.xerces.parsers.AbstractSAXParser.parse (Unknown source)

3 at org.xml.sax.helpers.XMLFilterImpl.parse (XMLFilterImpl.java $: 357$ )

Listing 8 An excerpt of the stack trace from the crash XRENDERING-422 with missing line numbers

\subsection{Missing Line Number}

31 frames in JCrashPack have frames with a missing line number, as shown in Listing 8. This happens if the Java files have been compiled without any debug information (by default, the Java compiler add information about the source files and line numbers, for instance, when printing a stack trace) or if the frame points to a class part of the standard Java library and the program has been run in the Java Runtime Environment (JRE) and not the JDK.

Since EvoCrash currently requires a line number to compute the fitness values during the search, those frames have been ignored during our evaluation and do not appear in the results. Yet, as frames with missing line number appear in JCrashPack (and in other stack traces), we decided to mention this trial here as a search-based crash reproduction challenge. A possible solution, as the future work, is to relax the fitness function so that it can still approximate fitness if line numbers are missing.

\section{Chosen Example XRENDERING-422. ${ }^{34}$}

\subsection{Incorrect Line Numbers}

In 37 cases, the target frame points to the line in the source code where the target class or method is defined. This happens when the previous frame points to an anonymous class or a lambda expression. Such frames practically cannot be used for crash reproduction as the location they point to does not reveal where exactly the target exception occurs. One possible solution would be to consider the frame as having a missing line number and use the relaxed fitness function to approximate the fitness.

Chosen Examples MATH-49b, frames 1 and $4 .^{35}$

\subsection{Unknown}

We were unable to identify why EvoCrash failed to reproduce 16 frames (out of 1,653 frames manually analyzed). In these cases, neither the logs nor the source code could help us understand how the exception was propagated.

Summary $\left(\mathrm{RQ}_{3}\right)$ What are the open problems that need to be solved to enhance searchbased crash reproduction? Based on the manual analysis of the frames that could not be reproduced at least once out of 10 rounds of executions, we identified 13 challenges

\footnotetext{
${ }^{34}$ The stack trace is available at https://github.com/STAMP-project/EvoCrash-JCrashPack-application/blob/ master/evaluation/JarFiles/resources/logs/XWIKI/XRENDERING-422/XRENDERING-422.log

${ }^{35}$ See https://github.com/STAMP-project/EvoCrash-JCrashPack-application/blob/master/results/examples/ IrrelevantFrames.md.
} 
for search-based crash reproduction. We confirmed challenges previously identified in other search-based software testing approaches and specified how they affect searchbased crash reproduction. And discovered new challenges, more specific to search-based crash reproduction and explained how the can affect other search-based software testing approaches.

These challenges are related to the difficulty to generate test cases due to complex input data, environmental dependencies, or complex code; abstraction (static initialization, interfaces, abstract, and anonymous classes); encapsulation mechanisms (private inner classes and nested private calls in the given stack trace) of object-oriented languages; or the selection of the target frame in crash reproduction (in try/catch blocks, in empty enumerations, when the location in the source code is unknown, or when the frame has an incorrect line number).

\section{Discussion}

\subsection{Empirical Evaluation for Crash Reproduction}

Conducting empirical evaluation for crash reproduction is challenging. It requires to collect various artifacts from different sources and to analyze the results to determine, in the case of a negative outcome, the cause that prevents the crash reproduction. Some are easy to fix, like missing dependencies that were added to the project linked to the stack trace, and for which we rerun the evaluation on the stack traces. The others are detailed in Section 8, and serve to identify future research directions.

One of the most surprising causes is due to a line mismatch in some stack traces. During the manual analysis of our results, we found out that three frames in two different stack traces, coming from Defects $4 \mathrm{~J}$ projects, target the wrong lines in the source code: the line numbers in the stack traces point to lines in the source code that cannot throw the targeted exception. Since the stack traces were collected directly from the Defects4J data (which reports failing tests and their outputs), we tried to regenerate them using the provided test suite and found a mismatch between the line numbers of the stack traces indeed. We reported those two projects to the Defects4J developers: ${ }^{36}$ a bug in JDK7 (2016) causes this mismatch. Since EvoCrash relies on line numbers to guide its search, it could not reproduce the crashes. We recompiled the source code, updated the stack trace accordingly in JCrashPack, and rerun the evaluation for those two stack traces.

Thanks to JCrashPack and ExRunner, we are now able to ease empirical evaluation for crash reproduction. ExRunner can be extended to other crash reproduction tools ${ }^{37}$ for comparison, or assess the development of new ideas in existing tools. Our future work also includes the prioritization of crashes from JCrashPack to allow quick feedback on new ideas in a fast and automated way (Alshahwan et al. 2018).

\subsection{Usefulness for Debugging}

In our evaluation, we focused on the crash-replication capabilities of EvoCrash and identified problems affecting those capabilities. We considered the generated tests only to classify

\footnotetext{
${ }^{36}$ See the issue at https://github.com/rjust/defects4j/issues/142.

${ }^{37}$ See how to extend ExRunner at https://github.com/STAMP-project/ExRunner.
} 
the outcomes of the EvoCrash generation process but did not assess their actual usefulness for debugging.

Chen and Kim (2015) introduced a usefulness criterion for the crash reproduction approaches. According to this criterion, a crash reproducing test is useful to the developers if it covers the buggy frame: i.e., if the target frame for which the reproduction is successful is higher than the frame that points to the buggy method.

In our previous work (Soltani et al. 2018), we conducted a controlled experiment to assess the usefulness of EvoCrash for debugging and bug fixing of two crashes (one from Apache Commons Collections and one from Apache Log4j) with 35 master students. Results show that using a crash-replicating test case generated by EvoCrash may help to locate and fix the defects faster. Also, this study confirmed the usefulness criterion defined by the Chen and Kim (2015) but also found evidence that test cases categorized as not useful can still help developers to fix the bug.

Since JCrashPack also includes two open source industrial and actively maintained applications, it represents an excellent opportunity to confirm the usefulness of EvoCrash in an industrial setting. The key idea is to centralize the information in the issue tracker by providing a test case able to replicate the crash reported in an issue in the same issue (as an attachment for instance). This can be automated using, for instance, a GitHub, GitLab or JIRA plugin that executes EvoCrash when a new issue contains a stack trace. To assess the usefulness of EvoCrash in an industrial setting, we plan to setup a case study (Wohlin et al. 2012) with our industrial partners. Hereafter, we outline the main steps of the evaluation protocol using XWiki as subject: (i) select four crashes to fix (two from open issues and two from closed issues) for which EvoCrash could generate a crash reproducing test for frame 3 or higher; (ii) clone the XWiki Git repository in GitHub and open four issues, corresponding to the four crash; (iii) remove the fix for the two fixed issues; (iv) for each issue, append the test case generated by EvoCrash; (v) ask (non-XWiki) developers to fix the issues; and finally, (vi) repeat the same steps without adding the test cases generated by EvoCrash (i.e., omit step iv). We would measure the time required to fix the issues (by asking participants to $\log$ that time). For the two previously fixed issues, we will compare the fixes provided by the participants with the fixes provided by XWiki developers. And for the two open issues, we will ask feedback from the XWiki developers through a pull request with the different solutions.

\subsection{Benchmark Building}

JCrashPack is the first benchmark dedicated to crash reproduction. We deliberately made a biased selection when choosing Elasticsearch as the most popular, trending, and frequentlyforked project from GitHub. Elasticsearch was among several other highly ranked projects, which addressed other application domains, and thus were interesting to explore. In the future, further effort should extend JCrashPack, possibly by: (i) using a random selection methodology for choosing projects; (ii) involving industrial projects from other application domains; and (iii) automatically collecting additional information about the crashes, the stack traces, and the frames to further understand current strengths and limitations of crash reproduction.

Building JCrashPack required substantial manual effort, not just for finding the issues, but also for collecting the right versions of the system itself and its dependencies needed to reproduce the given crash. Since we want it to be representative of current crashes, we need to automate this effort as much as possible: for instance, by mining stack traces from issue tracking systems (Nayrolles and Hamou-Lhadj 2016). 
Despite the benefits that the evaluation infrastructure could get from the inclusion of JCrashPack bugs in Defects4J, i.e., the isolation of the bugs to ease replicatbility of the evaluations (Just et al. 2014), we designed JCrashPack as a standalone instead of extending Defects4J. The main reason is that not all bugs in Defects4J manifest as crashes (only 73 out of 395 where selected to be part of JCrashPack). We also believe that the integration of the two benchmarks is not a smooth and easy process. Defects $4 \mathrm{~J}$ requires isolation of the buggy and fixed versions of the source code, as wel as a test case able to expose the bug (Just et al. 2014). However, not all issues were fixed at the time we collected the crashes in JCrashPack. Also, XWiki and Elasticsearch are much larger applications (124,000 NCSS for Elasticsearch, 177,000 NCSS for XWiki distributed in a hierarchy of several thousands of Maven projects) compared to the API libraries considered in Defects4J (63,000 NCSS for JFreeChart). Only building them with their default test suites already raised several issues. For those reasons, isolating the bug, the patch, and the non-regression test cases for such kind of large projects is not a trivial task.

\section{Future Research Directions for Search-Based Crash Reproduction}

From the evaluation and the challenges derived from our manual analysis, we devise the following future research directions. While the same challenge can be addressed in different ways, some requiring technical improvements of EvoCrash and other raising new research directions, we focus the discussion of this section on the latter.

\subsection{Context Matters}

While search-based crash-reproduction with EvoCrash (Soltani et al. 2017, 2018) outperformed other approaches based on (i) backward symbolic execution (Chen and Kim 2015), (ii) test case mutation (Xuan et al. 2015), and (iii) model-checking (Nayrolles et al. 2017), our evaluation shows that the extent to which crashes are reproduced varies. These results indicate the need for taking various types of contexts and properties of software applications into account when devising an approach to a problem. Thus, we show that indeed, rather than seeking a universal approach to search-based crash reproduction, it is important to find out and address challenges specific to various types of application domains (e.g., RESTful microservices vs. enterprise wiki applications) (Arcuri 2017).

Furthermore, search-based crash replication boils down to seeking the execution path that will reproduce a given stack trace. As with other search-based testing approaches, it faces challenges about input data generation during the search when the input space is large. Previous research on mocking and seeding (Arcuri et al. 2017; Rojas et al. 2016) address this problem by using functional mocking and extracting objects and constants from the bytecode.

We believe that taking context into account should go one step further for crash replication. With the development of DevOps (Roche 2013) and continuous integration and delivery pipelines, there is an increasing amount of available data on the execution of the software. Those data can be used to guide the search more accurately. For instance, by seeding the search using values observed in the execution logs and setting up values for environmental dependencies (databases, external services, etc.). 


\subsection{Stack Trace Preprocessing and Target Frame Selection}

Various factors may influence the selection of a target frame in a stack trace. As observed in our evaluation, when not performed cautiously, this selection leads to unsuccessful executions of EvoCrash. For instance, frames targeting code in a private inner class, or irrelevant source code location (like, as we observed, class header or annotation) should be discarded before performing the selection.

Frames targeting code in abstract classes or interfaces (only if the target method is defined in the interface, which is possible from Java 8) may be of some use to find the cause of the crash: for instance, to identify an incorrect subclass implementation (Liskov and Guttag 2000). However, as abstract classes and interfaces cannot be directly instantiated, the stack trace generated by EvoCrash can never be exactly the same as the given stack trace. And, as for input arguments and generic type parameters, EvoCrash has no indication on which subclass to pick, making the search difficult. In this case, considering higher level frames (i.e., frames that are lower in the stack trace) may help to pick the right subclass.

Those reasons motivate the need to develop stack trace analysis techniques in order to help the selection of a target frame. This analysis will discard irrelevant and unknown source location frames and provide a visualization to the developer to have a clear view on what are his or her options, for instance by marking stack traces that point to interfaces and abstract classes and recommend him to pick higher level frames.

For a given stack trace, this analysis will also identify frames pointing to a try/catch block. Those stack traces are commonly reported by users to issue tracking systems but cannot (for now) be completely reproduced by EvoCrash. Further investigation on current error handling practices in Java code (Cabral and Marques 2007; Coelho et al. 2017) and how they are reported by users (Maiga et al. 2015) will help us to devise efficient approaches to replicate such stack traces.

\subsection{Guided Search}

Besides usage of contextual information to enhance the generation of test cases during the search process, we also consider to enhance the guidance itself. Search based testing algorithms have several parameters (365 in EvoCrash), like population size, search budget, probability of applying crossover and mutation, etc. As demonstrated by Arcuri and Fraser (2011), default parameters values work well on average, but may be fare from optimal for specific frames and stack traces. A better characterization of the stack traces in JCrashPack, trying different parameters, as well as improving the fitness function itself are part of our future work. For instance the fitness function could take other elements into account (e.g., compute a similarity for exception messages). We will also consider multi-objectives search, where, for a given target frame, reproducing each lower frame becomes an objective of the search. We plan to reuse our evaluation infrastructure to compare those different approaches and investigate their different fitness landscapes to gain deeper understanding of the search process for crash reproduction. And eventually devise guidelines on EvoCrash settings to maximize crash reproduction for a given stack trace and its characteristics.

\subsection{Improving Testability}

Finally, as we observed, code complexity was among the major challenges in crash reproduction with EvoCrash. To improve testability, several testability transformation techniques 
(McMinn 2011; Li and Fraser 2011; Harman et al. 2004; Harman et al. 2002; Baresel et al. 2004) have been proposed in the literature so far. Future research may investigate testability transformation techniques and their impact on search-based crash reproduction.

\section{Threats to Validity}

Evaluations of crash reproduction approaches, such as the one we conducted for EvoCrash, come with threats to internal validity, external validity, and reliability. The overarching goal of JCrashPack is to reduce such threats for all evaluations of any crash reproduction tool, by offering a curated set of crashes to conduct such evaluations.

Concerning external validity, we carefully designed JCrashPack so that it offers a mix of small and large systems, as well as of different types of exceptions. Furthermore, it includes open source systems directly developed by industry. Nevertheless, any set is incomplete, which is why we keep JCrashPack open for extension, as discussed in Section 9. For example, there still remain several other domains, such as gaming or financial applications, for which there is no representative project in the benchmark.

With respect to internal validity, implementation faults can be a source of confounding factors. These can occur in the tools themselves, such as EvoCrash or EvoSuite, but also in the infrastructure used to actually conduct the experiment. To address the latter, JCrashPack comes with ExRunner, which automates the process of scheduling, executing, monitoring, and reporting crash reproduction attempts.

Concerning reliability, JCrashPack and ExRunner make it easy to repeat experiments, thus making it possible for researchers to independently replicate each others crash reproduction findings.

Besides these threats partially mitigated by JCrashPack, our evaluation of EvoCrash comes with additional threats to (internal and external) validity. This particularly relates to the randomized nature of genetic algorithms, which we addressed by running the evaluations 10 times, and following the guidelines by Arcuri and Briand (2014) for analyzing the results. Furthermore, such threats concern the risk of bias during the manual analysis, which we mitigated by using cross-checking: the result of each manual analysis has been validated by at least one other person. In case of disagreement, we asked for a third opinion. Finally, our evaluation includes only one tool: EvoCrash. Previous work showed that EvoCrash performs better than other state-of-the-art crash reproduction tools. Unfortunately, since to the best of our knowledge, no other tool was publicly available, we were not able to confirm that conclusion on the crashes in JCrashPack. We believe that JCrashPack enhances the current state-of-the-practice in crash reproduction research by offering a publicly available benchmark for which other tool providers can report their results.

\section{Conclusion}

Experimental evaluation of crash reproduction research is challenging, due to the computational resources needed by reproduction tools, the difficulty of finding suitable real life crashes, and the intricacies of executing a complex system so that the crash can be reproduced at all.

To remedy this problem, this paper sets out to create a benchmark of Java crashes, that can be reused for experimental purposes. To that end we propose JCrashPack and ExRunner, 
a curated benchmark of 200 real life crashes, and a tool to conduct massive experiments on these crashes. This benchmark is publicly available and can be used to compare existing and new tools against each other, as well as to analyze how proposed improvements to existing reproduction techniques actually constitute an improvement.

We applied the state of the art search-based Java crash reproduction tool, EvoCrash, to JCrashPack. Our findings include that the state of the art can reproduce 87 crashes out of 200 in a majority of time, that crash reproduction for industry-strength systems is substantially harder, and that NullPointerExceptions are generally easiest to reproduce. Furthermore, we identified 13 challenges that crash reproduction research needs to address to strengthen uptake in practice, as well a future research directions to address those challenges.

JCrashPack can be extended in various ways: by including more crashes from other types of applications; by automating the collection of information about eh crashes and stack traces to further understand current strengths and limitations of crash reproduction; as well as automating the collection of the crashes themselves. Furthermore, since executing crash reproduction tools on 200 crashes may be time taking, JCrashPack could be extended to offer prioritization for benchmarks, based on the known theoretical strengths and limitations if the tools. For instance, by ordering crashes based on the cyclomatic complexity of the involved frames to evaluate search-based or symbolic execution-based crash reproduction approaches.

Finally, our future work for EvoCrash itself include improving input data generation by taking information from the execution context and the application (e.g., existing source code and test cases) into account. We also want to deeper our understanding of stack traces in order to be able to recommend target frames to the developers. Finally, we will improve the search process itself by refining the fitness function to improve the guidance trough the different frames of the stack trace.

Acknowledgements We especially thank Danny Groenewegen for the technical expertise and infrastructure he provided during the initial evaluations. We would also like to thank Andy Zaidman, Oscar Luis Vera Pérez, Gilles Perrouin, and Joseph Hejderup for their feedback on earlier versions of the paper. This research was partially funded by the EU Project STAMP ICT-16-10 No.731529 and the NIRICT 3TU.BSR (Big Software on the Run) project.

Open Access This article is distributed under the terms of the Creative Commons Attribution 4.0 International License (http://creativecommons.org/licenses/by/4.0/), which permits unrestricted use, distribution, and reproduction in any medium, provided you give appropriate credit to the original author(s) and the source, provide a link to the Creative Commons license, and indicate if changes were made.

\section{References}

Alshahwan N, Gao X, Harman M, Jia Y, Mao K, Mols A, Tei T, Zorin I (2018) Deploying search based software engineering with Sapienz at Facebook. In: Search-based software engineering. SSBSE 2018, LNCS, vol 11036. Springer. https://doi.org/10.1007/978-3-319-99241-9_1

Apache (2017) Ant. http://ant.apache.org/. [Online; Accessed 25 Jan 2018]

Apache (2017) Commons Collections. https://commons.apache.org/proper/commons-collections/. [Online; Accessed 25 Jan 2018]

Apache (2017) Log4j. https://logging.apache.org/log4j/2.x/. [Online; Accessed 25 Jan 2018]

Arcuri A (2017) RESTful API automated test case generation. In: 2017 IEEE international conference on software quality, reliability and security (QRS). IEEE, pp 9-20. https://doi.org/10.1109/QRS.2017.11

Arcuri A, Briand L (2014) A hitchhiker's guide to statistical tests for assessing randomized algorithms in software engineering. Software Testing, Verification and Reliability 24(3):219-250 
Arcuri A, Fraser G (2011) On parameter tuning in search based software engineering. In: Population english edition, pp 33-47. https://doi.org/10.1007/978-3-642-23716-4_6

Arcuri A, Fraser G, Galeotti JP (2014) Automated unit test generation for classes with environment dependencies. In: Proceedings of the 29th ACM/IEEE international conference on Automated software engineering - ASE'14. ACM Press, Vasteras, pp 79-90. https://doi.org/10.1145/2642937.2642986

Arcuri A, Fraser G, Just R (2017) Private api access and functional mocking in automated unit test generation. In: 2017 IEEE international conference on software testing, verification and validation (ICST). IEEE, IEEE Computer Society, Tokyo, pp 126-137

Artzi S, Kim S, Ernst MD (2008) Recrash: Making software failures reproducible by preserving object states. In: Proceedings of the 22Nd European Conference on Object-Oriented Programming, ECOOP '08. Springer-Verlag, Berlin, Heidelberg, pp 542-565. https://doi.org/10.1007/978-3-540-70592-5_23

Baresel A, Binkley D, Harman M, Korel B (2004) Evolutionary testing in the presence of loop-assigned flags: a testability transformation approach. In: ACM SIGSOFT software engineering notes, vol 29. ACM, ACM, Boston, pp 108-118

Bell J, Sarda N, Kaiser G (2013) Chronicler: lightweight recording to reproduce field failures. In: Proceedings of the 2013 international conference on software engineering, ICSE '13. IEEE Press, Piscataway, pp 362-371

Bianchi FA, Pezzè M, Terragni V (2017) Reproducing concurrency failures from crash stacks. In: Proceedings of the 2017 11th joint meeting on foundations of software engineering. ACM, ACM, Paderborn, pp 705-716

Braione P, Denaro G, Mattavelli A, Pezzè M (2017) Combining symbolic execution and search-based testing for programs with complex heap inputs. In: Proceedings of the 26th ACM SIGSOFT international symposium on software testing and analysis, ISSTA 2017. ACM, New York, pp 90-101. https://doi.org/10.1145/3092703.3092715

Briand L, Bianculli D, Nejati S, Pastore F, Sabetzadeh M (2017) The case for context-driven software engineering research: generalizability is overrated. IEEE Softw 34(5):72-75. https://doi.org/10.1109/MS. 2017.3571562

Cabral B, Marques P (2007) Exception handling: a field study in java and .NET. In: ECOOP 2007 - Objectoriented programming, vol 4609. Springer, Berlin, pp 151-175. https://doi.org/10.1007/978-3-54073589-2_8

Cao Y, Zhang H, Ding S (2014) Symcrash: selective recording for reproducing crashes. In: Proceedings of the 29th ACM/IEEE international conference on automated software engineering, ASE '14. ACM, pp 791-802. https://doi.org/10.1145/2642937.2642993

Chen N, Kim S (2015) STAR: stack trace based automatic crash reproduction via symbolic execution. IEEE Trans Softw Eng 41(2):198-220. https://doi.org/10.1109/TSE.2014.2363469

Clause J, Orso A (2007) A technique for enabling and supporting debugging of field failures. In: Proceedings of the 29th international conference on software engineering, ICSE '07. IEEE Computer Society, Washington, pp 261-270. https://doi.org/10.1109/ICSE.2007.10

Coelho R, Almeida L, Gousios G, van Deursen A, Treude C (2017) Exception handling bug hazards in Android. Empir Softw Eng 22(3):1264-1304. https://doi.org/10.1007/s10664-016-9443-7

Dubbo (2018) A high-performance, java based, open source RPC framework. http://dubbo.io. [Online; Accessed 25 Jan 2018]

Elastic (2018) Elasticsearch: RESTful, Distributed Search and Analytics. https:/www.elastic.co/products/ elasticsearch. [Online; Accessed 25 Jan 2018]

Fraser G, Arcuri A (2013) Computer Society. In: 2013 IEEE sixth international conference on software testing, verification and validation (ICST). IEEE, IEEE, Luxembourg, pp 362-369

Fraser G, Arcuri A (2013) Whole test suite generation. IEEE Trans Softw Eng 39(2):276-291

Fraser G, Arcuri A (2014) Automated test generation for java generics. In: International conference on software quality. Springer, Vienna, pp 185-198

Fraser G, Arcuri A (2014) A large-scale evaluation of automated unit test generation using evosuite. ACM Trans Soft Eng Methodol (TOSEM) 24(2):8

Gómez M, Rouvoy R, Adams B, Seinturier L (2016) Reproducing context-sensitive crashes of mobile apps using crowdsourced monitoring. In: Proceedings of the Int Conf Mobile Soft Eng Syst, MOBILESoft '16. ACM, New York, pp 88-99. https://doi.org/10.1145/2897073.2897088

Harman M, Hu L, Hierons R, Baresel A, Sthamer H (2002) Improving evolutionary testing by flag removal. In: Proceedings of the 4th annual conference on genetic and evolutionary computation. Morgan Kaufmann Publishers Inc., Morgan Kaufmann, New York, pp 1359-1366

Harman M, Hu L, Hierons R, Wegener J, Sthamer H, Baresel A, Roper M (2004) Testability transformation. IEEE Trans Softw Eng 30(1):3-16 
Harman M, Mansouri SA, Zhang Y (2012) Search-based software engineering: trends, techniques and applications. ACM Comput Surv (CSUR) 45(1):11

Java Design Patterns (2018) Design patterns implemented in Java. http://java-design-patterns.com. [Online; Accessed 25 Jan 2018]

JDK (2016) Stack trace has invalid line numbers. https://bugs.openjdk.java.net/browse/JDK-7024096. [Online; Accessed 25 Jan 2018]

Jin W, Orso A (2012) Bugredux: reproducing field failures for in-house debugging. In: Proceedings of the 34th international conference on software engineering, ICSE '12. IEEE Press, Piscataway, pp 474-484

Just R, Jalali D, Ernst MD (2014) Defects4J: a database of existing faults to enable controlled testing studies for Java programs. In: Proceedings of the 2014 international symposium on software testing and analysis - ISSTA 2014. ACM Press, San Jose, pp 437-440. https://doi.org/10.1145/2610384.2628055

Kifetew FM, Jin W, Tiella R, Orso A, Tonella P (2013) Sbfr: a search based approach for reproducing failures of programs with grammar based input. In: Proceedings of the 28th IEEE/ACM international conference on automated software engineering, ASE'13. IEEE Press, Piscataway, pp 604-609. https://doi.org/10.1109/ASE.2013.6693120

Kifetew FM, Jin W, Tiella R, Orso A, Tonella P (2014) Reproducing field failures for programs with complex grammar-based input. In: Proceedings of the 2014 IEEE international conference on software testing, verification, and validation, ICST '14. IEEE Computer Society, Washington, pp 163-172. https://doi.org/10.1109/ICST.2014.29

Leitner A, Ciupa I, Oriol M, Meyer B, Fiva A (2007) Contract driven development $=$ test driven development - writing test cases. In: Proceedings of the the 6th joint meeting of the european software engineering conference and the ACM SIGSOFT symposium on the foundations of software engineering, ESEC-FSE '07. ACM, New York, pp 425-434. https://doi.org/10.1145/1287624.1287685

Leitner A, Pretschner A, Mori S, Meyer B, Oriol M (2009) On the effectiveness of test extraction without overhead. In: 2009 International conference on software testing verification and validation. IEEE, pp 416-425. https://doi.org/10.1109/ICST.2009.30

Li Y, Fraser G (2011) Bytecode testability transformation. In: International symposium on search based software engineering. Springer, Szeged, pp 237-251

Liskov B, Guttag J (2000) Program development in JAVA: abstraction, specification, and object-oriented design. Pearson Education, London

Luo Q, Hariri F, Eloussi L, Marinov D (2014) An empirical analysis of flaky tests. In: Proceedings of the 22nd ACM SIGSOFT international symposium on foundations of software engineering - FSE 2014, pp 643-653. https://doi.org/10.1145/2635868.2635920

Maiga A, Hamou-Lhadj A, Nayrolles M, Koochekian Sabor K, Larsson A (2015) An empirical study on the handling of crash reports in a large software company: an experience report. In: 2015 IEEE International conference on software maintenance and evolution (ICSME). IEEE, Bremen, pp 342-351. https://doi.org/10.1109/ICSM.2015.7332485

Malburg J, Fraser G (2011) Combining search-based and constraint-based testing. In: 2011 26th IEEE/ACM international conference on automated software engineering (ASE). IEEE, IEEE Computer Society, Lawrence, pp 436-439

McMinn P (2004) Search-based software test data generation: a survey. Software Testing, Verification and Reliability 14(2):105-156. https://doi.org/10.1002/stvr.294

McMinn P (2011) Search-based software testing: past, present and future. In: 2011 ieee fourth international conference on software testing, verification and validation workshops (icstw). IEEE, IEEE Computer Society, Berlin, pp 153-163

Narayanasamy S, Pokam G, Calder B (2005) Bugnet: continuously recording program execution for deterministic replay debugging. In: Proceedings of the $32 \mathrm{Nd}$ annual international symposium on computer architecture, ISCA '05. IEEE Computer Society, Washington, pp 284-295. https://doi.org/10.1109/ISCA. 2005.16

Nayrolles M, Hamou-Lhadj A (2016) BUMPER: a tool for coping with natural language searches of millions of bugs and fixes. In: 2016 IEEE 23Rd international conference on software analysis, evolution, and reengineering (SANER). IEEE, Suita, pp 649-652. https://doi.org/10.1109/SANER.2016.71

Nayrolles M, Hamou-Lhadj A, Tahar S, Larsson A (2017) A bug reproduction approach based on directed model checking and crash traces. Journal of Software: Evolution and Process 29(3):e1789. https://doi.org/10.1002/smr.1789

Oracle (2019) What's new in JDK 8. https://www.oracle.com/technetwork/java/javase/8-whats-new-2157071. html. Accessed: 14 May 2019

Roche J (2013) Adopting DevOps practices in quality assurance. Commun ACM 56(11):38-43. https://doi.org/10.1145/2524713.2524721 
Rojas JM, Fraser G, Arcuri A (2016) Seeding strategies in search-based unit test generation. Software Testing, Verification and Reliability 26(5):366-401. https://doi.org/10.1002/stvr.1601

Rößler J, Zeller A, Fraser G, Zamfir C, Candea G (2013) Reconstructing core dumps. In: Proceedings IEEE 6th international conference on software testing, verification and validation, ICST 2013. IEEE, pp 114-123. https://doi.org/10.1109/ICST.2013.18

RxJava (2018) Reactive Extensions for the JVM. https://github.com/ReactiveX/RxJava. [Online; Accessed 25 Jan 2018]

Sim SE, Easterbrook S, Holt RC (2003) Using benchmarking to advance research: a challenge to software engineering. In: Proceedings of the 25th International Conference on Software Engineering, ICSE ' 03. IEEE Computer Society, Portland, pp 74-83

Soltani M, Panichella A, Van Deursen A (2017) A guided genetic algorithm for automated crash reproduction. In: 2017 IEEE/ACM 39Th international conference on software engineering (ICSE). IEEE, Buenos Aires, pp 209-220. https://doi.org/10.1109/ICSE.2017.27

Soltani M, Panichella A, van Deursen A (2018) Search-based crash reproduction and its impact on debugging. IEEE Trans Softw Eng. https://doi.org/10.1109/TSE.2018.2877664

Steven J, Chandra P, Fleck B (2000) Podgurski, a.: jrapture: a capture/replay tool for observation-based testing. In: Proceedings of the 2000 ACM SIGSOFT international symposium on software testing and analysis, ISSTA '00. ACM, New York, pp 158-167. https://doi.org/10.1145/347324.348993

Weeratunge D, Zhang X, Jagannathan S (2010) Analyzing multicore dumps to facilitate concurrency bug reproduction. In: Proceedings of the fifteenth edition of ASPLOS on architectural support for programming languages and operating systems, ASPLOS XV. ACM, New York, pp 155-166. https://doi.org/10.1145/1736020.1736039

Wohlin C, Runeson P, Höst M, Ohlsson MC, Regnell B, Wesslén A (2012) Experimentation in software engineering. Springer, Berlin. https://doi.org/10.1007/978-3-642-29044-2

Xiao X, Xie T, Tillmann N, De Halleux J (2011) Precise identification of problems for structural test generation. In: 2011 33rd international conference on software engineering (ICSE). IEEE, ACM, Honolulu, pp 611-620

Xuan J, Xie X, Monperrus M (2015) Crash reproduction via test case mutation: let existing test cases help. In: Proceedings of the 2015 10th joint meeting on foundations of software engineering - ESEC/FSE 2015. ACM Press, New York, pp 910-913. https://doi.org/10.1145/2786805.2803206

XWiki (2018) The advanced open source enterprise and application Wiki. http://www.xwiki.org/. [Online; Accessed 25 Jan 2018]

Yu T, Zaman TS, Wang C (2017) Descry: reproducing system-level concurrency failures. In: Proceedings of the 2017 11th joint meeting on foundations of software engineering. ACM, pp 694-704

Zamfir C, Candea G (2010) Execution synthesis: a technique for automated software debugging. In: Proceedings of the 5th European conference on computer systems, EuroSys '10. ACM, New York, pp 321-334. https://doi.org/10.1145/1755913.1755946

Zeller A (2009) Why programs fail - a guide to systematic debugging, 2nd edn. Academic Press, New York

Publisher's note Springer Nature remains neutral with regard to jurisdictional claims in published maps and institutional affiliations.

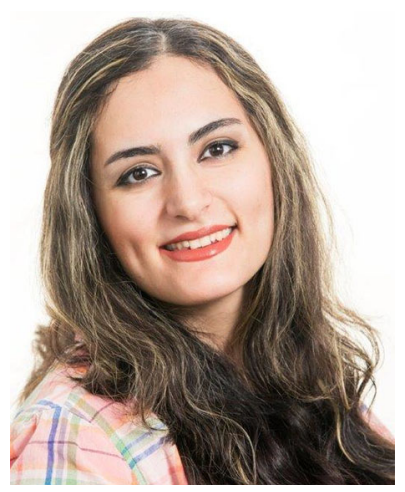

Mozhan Soltani is a PhD student in the Programming Education Research Lab (PERL) at Leiden University in the Netherlands. She has a broad range of interests in the field of software engineering, including development and verification of programming languages, software architecture, and software development processes. She has served as a reviewer of the journal of Software Testing, Verification and Reliability (STVR) and as program committee of the MSR'18 Mining Challenge. 


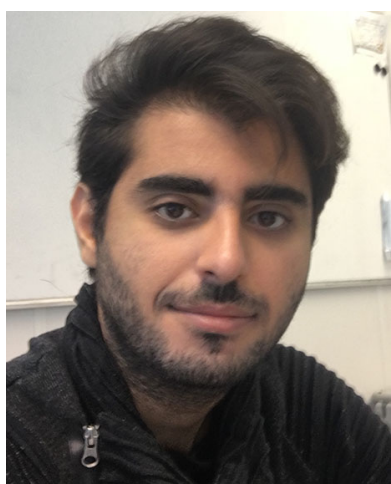

Pouria Derakhshanfar is a Ph.D. student in the software engineering research group (SERG) of the Delft University of Technology. He started his Ph.D. in 2017 under the supervision of Prof. Andy Zaidman and Prof. Arie van Deursen. He is part of the EU Software Testing AMPlification (STAMP) project, where he develops new search-based approaches for crash replication and test amplification in a DevOps context. His research interests include search-based software testing, model-based testing, and log analysis.

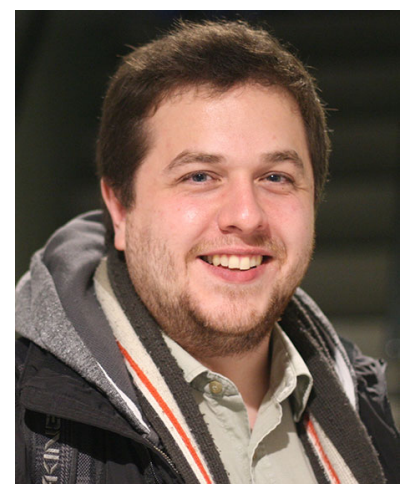

Xavier Devroey is a post-doctoral researcher in the software engineering research group (SERG) of the Delft University of Technology, where he is involved in the EU Software Testing AMPlification (STAMP) project. He received his Ph.D. in Computer Science from the University of Namur. His main research interests are searchbased and model-based software testing, test suite augmentation, and variability-intensive systems engineering.

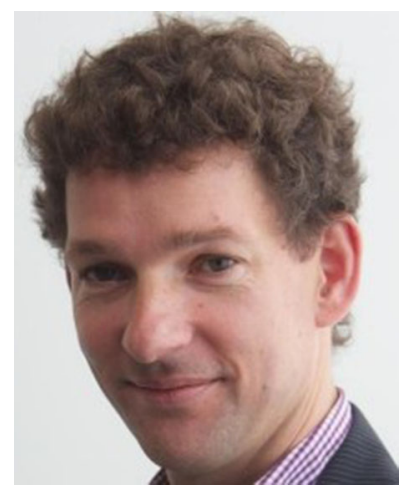

Arie van Deursen is a professor in software engineering at Delft University of Technology, where he chairs the Department of Software Technology. His research interests include test amplification, software analytics, AI-based software engineering, continuous delivery, and human aspects of software development. He was program co-chair of ESEC/FSE 2017, and will be program co-chair of ICSE 2021. He serves on the steering committees of the ESEC/FSE and ICSE conference series. 


\section{Affiliations}

Mozhan Soltani $^{1,2} \cdot$ Pouria Derakhshanfar $^{1} \cdot$ Xavier Devroey $^{1}$ (D) Arie van Deursen ${ }^{1}$

Mozhan Soltani

m.soltani@tudelft.nl

Pouria Derakhshanfar

p.derakhshanfar@tudelft.nl

Arie van Deursen

arie.vandeursen@tudelft.nl

1 Delft University of Technology, Postbus 5, Delft, 2600 AA, The Netherlands

2 Present address: Leiden University, Rapenburg 70, Leiden, 2311 EZ, The Netherlands 